\title{
PREDICTIVE RELAY-SELECTION COOPERATIVE DIVERSITY IN LAND MOBILE SATELLITE SYSTEMS
}

\author{
Babatunde Awoyemi $^{1 *}$, Tom Walingo ${ }^{1}$, Fambirai Takawira ${ }^{2}$ \\ ${ }^{1}$ Department of Electronic Engineering, University of KwaZulu-Natal, South Africa \\ ${ }^{2}$ School of Electrical and Information Engineering, University of Witwatersrand, South Africa \\ *Correspondence to: Babatunde Awoyemi, Department of Electronic Engineering, University of Pretoria, South \\ Africa. \\ E-mail: awoyemibabatunde@gmail.com
}

Abstract - Cooperative diversity protocols promise a new dimension of diversity that provides better communication by engaging nearby relays in forming a 'virtual' array of antennas for combined signal transmission. The current incremental cooperative diversity algorithms incrementally select best relay(s) to cooperate based on the channel quality reported by the relays. However, the algorithms do not take into consideration the fact that the chosen best relay(s) at estimation may not always be best at the time of communication. This is due to the time delay between the relay selection and its transmission of signal (problem of outdated Channel Quality Information). To solve this problem, the concept of channel prediction is introduced and employed whereby each relay determines a predicted value of its Channel Quality Information (CQI) based on its past measurements. The paper therefore develops a novel predictive relay-selection (PRS) cooperative diversity model which seeks to improve Land Mobile Satellite (LMS) communication through prediction protocols. In the model, the chosen best relay is the one with the best predicted CQI value instead of the traditional outdated one. Performance analysis of outage probability and average bit error probability for the newly developed PRS cooperation shows that the PRS cooperation is better than direct and outdated CQI relay communication.

Keywords - Channel Quality Information, Cooperative Diversity, Incremental Relaying, Land Mobile Satellite, Prediction Algorithms

\section{INTRODUCTION}

Cooperative diversity protocols promise a new dimension of diversity that reliably provides better communication by engaging nearby relays for combined signal transmission or reception. In cooperative diversity, the various transmitting antennas (in transmitter-based cooperation) or receiving antennas (in receiver-based cooperation) of nearby relays are employed in forming a 'virtual array' of antennas thus achieving the diversity advantage [1]. Receiver-based cooperative diversity can be applied to the Land Mobile Satellite (LMS) systems' downlink to improve communication. Receiver-based cooperative diversity can be classified according to the cooperating relay node behavior before forwarding its received signal e.g. Amplify-and-Forward (AF) [2], Decode-and-Forward (DF) [3] and coded cooperation (CC) [4] and by the various schemes for selecting the cooperating relay nodes 
e.g. Single Relay Selection (SRS) [5], Multiple Relay Selection (MRS) [6], Incremental Relay Selection (IRS) [7], and All-Relay Participation [8]. In this paper, both classification types are considered for the LMS system.

While the receiver-based cooperative diversity concept has been adequately investigated in terrestrial networks, the applicability of the current schemes to LMS systems is seriously undermined. This could be due to the fact that the relay nodes which act as cooperators are currently sparse over a large area distribution. The introduction of hybrid satellite-terrestrial networking is currently being developed, meaning that more satellite mobile terminals will be readily available in the near future, thus enhancing the feasibility. However, a general problem with receiver-based cooperative diversity using relay selection is the problem of outdated channel quality information (CQI). The problem arises from the fact that the relay nodes are mobile and their channel gains vary with time. Since there is usually a propagation delay between the time of estimation and time of transmission of signal by the relays, the CQI on which the relays are selected is therefore imperfect or outdated [9]. The outdated CQI problem is even more exacerbated in the LMS system because of the long propagation delay (about 250ms for Geostationary Earth Orbit (GEO) satellites) between the satellite transmission time and the relays/destination reception time for a return link. The outdated CQI problem is thus a serious challenge for cooperative diversity in LMS systems.

Investigations into the effect of outdated CQI on the performance of cooperative diversity are currently underway. In [9] and [10], the viability and gains of using decode-and-forward cooperative diversity in a wireless terrestrial network (e.g. WiMAX) using opportunistic relay selection scheme with outdated CQI is investigated. It is shown that the opportunistic relaying cooperation experiences a performance loss as well as a diversity loss when the CQI is not exact and when the number of relays available for cooperation is low. In [11], relay selection in amplify-and-forward cooperative diversity with outdated CQI is addressed. A notable deduction is that if the correlation coefficient of the CQI at estimation and its value at transmission is not unity (outdated CQI problem) there is a significant performance loss in the cooperative protocol. Furthermore the diversity order of all single 'best' relay selection schemes which would have achieved full diversity in the presence of a perfect CQI reduces to unity in the presence of outdated CQI [12] - [14]. Generally, the diversity order of all relayselection cooperative diversity schemes reduces to unity in the face of outdated CQI. To guarantee a better performance through relay-selection cooperation, the problem of outdated CQI must be adequately combated.

There are currently proposed solutions to the outdated CQI challenge on cooperative diversity based networks. These include; the use of multiple relays instead of a single relay [15], using additional private messages with the originally sent signal [16], the use of an average SNR criterion in designing an optimal combination of beamformers [17] and the use of multiple estimates of signal quality from different frequencies or times [18]. The signal quality estimation in [18] was carried out using the Mean Square Error (MSE) technique while the estimation error was characterized as additive Gaussian noise. Generally, the investigated solutions are applicable to cooperative diversity in terrestrial networks and might not be exactly applicable to the satellite system, given its characteristically long propagation delay. 
As a solution to the outdated CQI problem for receiver-based cooperative diversity in LMS systems, we propose and investigate predictive relay-selection (PRS) cooperation. The PRS cooperative diversity concept makes use of prediction algorithms in determining future CQI of the relay links thus mitigating the outdated CQI challenge. An appropriate LMS fading model is used in the cooperative diversity investigation for a better and more accurate network performance. The main contributions of this work are;

- Developing and analyzing a novel predictive relay-selection (PRS) cooperative diversity model for Land Mobile Satellite Systems (LMS) systems.

- Application of linear prediction [19], [20] and pattern-matching prediction [23] algorithms to the PRS cooperative diversity in Land Mobile Satellite systems.

The remaining parts of this paper are organized as follows. In Section II, the PRS cooperative diversity model for LMS is developed. Section III discusses the various prediction models that are applied to the LMS. Section IV has the analysis of the performance metrics considered for the PRS cooperation. The results of the PRS cooperative diversity scheme (in comparison with direct communication and cooperation with outdated CQI) are discussed in Section V and the conclusions given in Section VI.

\section{PREDICTIVE RELAY-SELECTION COOPERATIVE DIVERSITY CHANNEL MODEL}

\section{Network Model}

The modeled network is the downlink receiver-based cooperative diversity satellite channel as shown in Fig. 1. It consist of one satellite $(S)$, one destination $(D)$ and $R_{1}$ to $R_{N}$ cooperative relays out of which a best relay $\left(R_{B}\right)$ will be selected to cooperate. The communication is incremental. The algorithm checks the state of the direct channel, if good, direct communication is employed if not cooperative communication is employed. The channel state (good, bad) depends on a threshold CQI (SNR) value. The signal transmission (total frame) is in two time slots (see Fig. 2. and Fig. 3.) for direct and cooperative communications respectively. In the first slot, the satellite $(\mathrm{S})$ broadcasts its signals to both the destination terminal (D) and the relays $\left(R_{N}\right)$. The destination terminal checks the received CQI and compares it to the threshold CQI - a positive acknowledgement (ACK) of received signal is broadcasted by the destination to both satellite and all relays after every time slot indicating a successful transmission. The relays are not employed in that time slot. The satellite sends its next signal in the second time slot. However, if the received CQI at the destination is below the threshold CQI, a negative acknowledgement (NACK) of received signal is broadcasted by the destination to the satellite and the relays indicating a bad link hence unsuccessful transmission. The satellite does not send a signal in the second time slot. Each relay calculates its predicted Channel Quality Information (CQI) using the prediction algorithms and sends this to the destination. The destination selects the terminal with the 


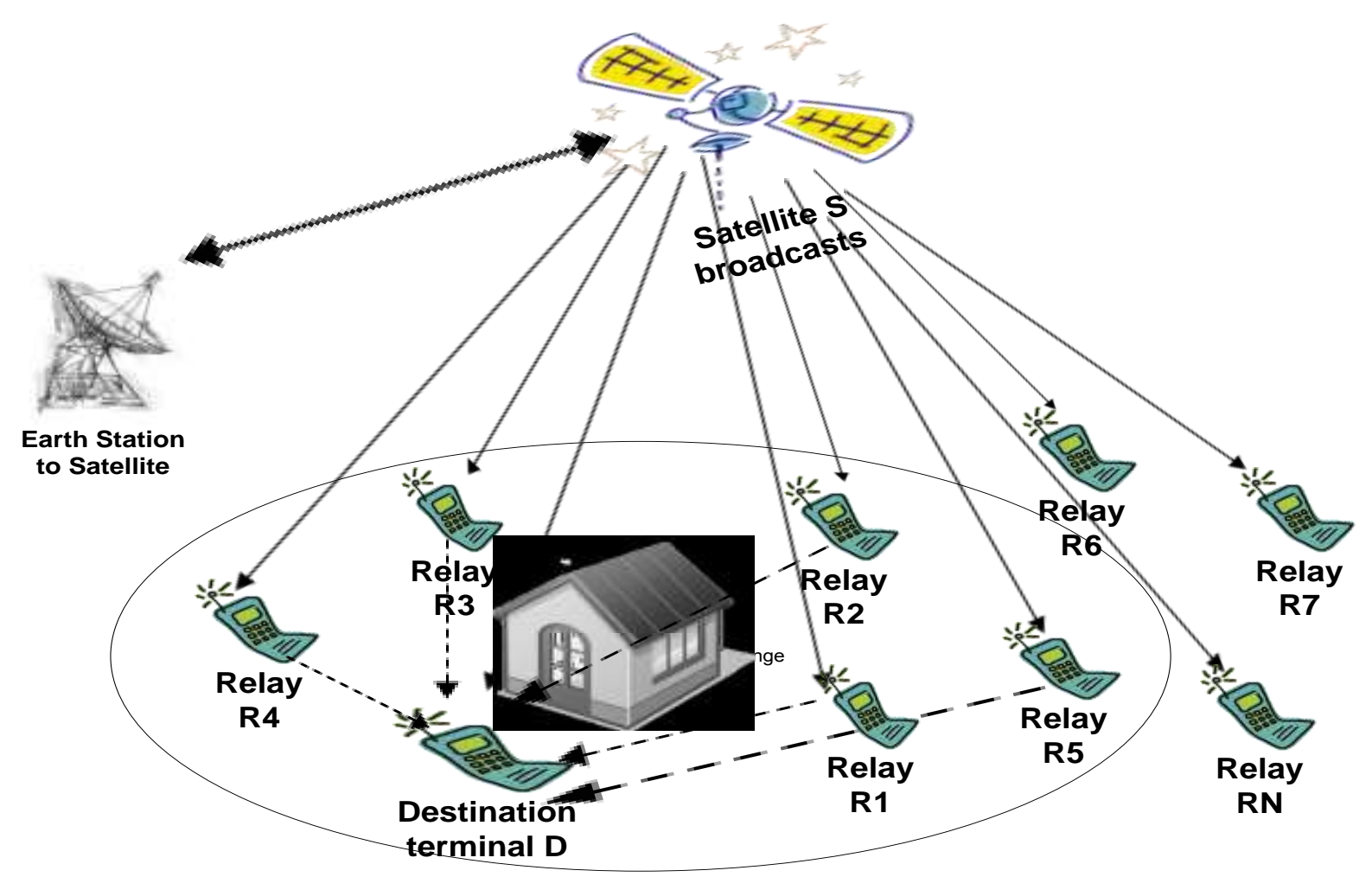

Fig. 1 Receiver-based cooperative diversity for land mobile satellite system

Total Frame for Direct Transmission

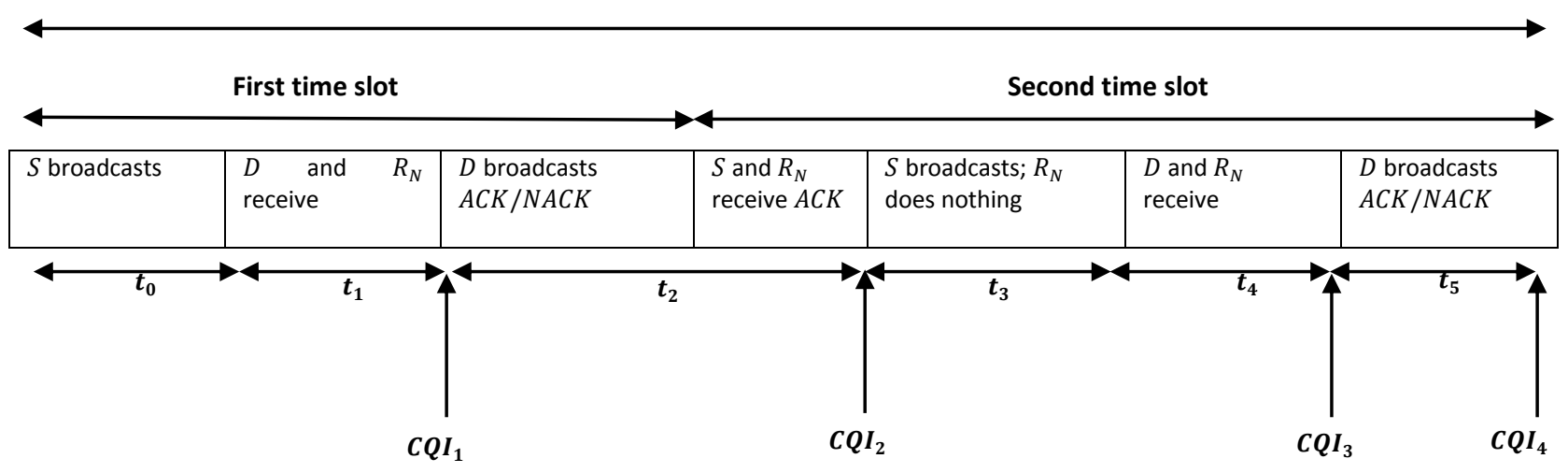

Fig. 2. Total Time Frame for Direct Communication

Total Frame for Predictive Cooperative Transmission

\begin{tabular}{|c|c|c|c|c|c|c|c|}
\hline \multicolumn{3}{|c|}{ First time slot } & \multicolumn{5}{|c|}{ Second time slot } \\
\hline$S$ broadcasts & $\begin{array}{l}D \text { and } R_{N} \\
\text { receives }\end{array}$ & $\begin{array}{l}D \text { broadcasts } \\
A C K / N A C K\end{array}$ & $\begin{array}{l}S \quad \text { and } R_{N} \\
\text { receive } N A C K \text {. } \\
S \text { waits }\end{array}$ & $\begin{array}{c}R_{N} \text { predicts } \\
C Q I_{5}\end{array}$ & $\begin{array}{l}R_{N} \text { send } C Q I_{2} \\
\text { and } C Q I_{5} \\
\text { to } D\end{array}$ & $\begin{array}{l}D \text { sends a CBR } \\
\text { to } R_{B} \text { with } \\
\text { best } C Q I\end{array}$ & $\begin{array}{ll}R_{B} & \text { sends } \\
\text { signal } & \end{array}$ \\
\hline \multicolumn{8}{|l|}{$t_{0}$} \\
\hline & $C Q$ & & $C Q$ & & & & $I_{5}$ \\
\hline
\end{tabular}

Fig. 3. Total Time Frame for Cooperative Communication 
highest predicted CQI (i.e. selected best relay $R_{B}$ ) as the cooperative relay. Next, the destination terminal broadcasts a single bit CBR (Chosen Best Relay) signal containing the identity of the selected best relay for communication. Relays not selected ignore the CBR. The selected best relay enters into a transmitting mode after receiving the CBR. It forwards its received signal to the destination. The best relay's signal is afterwards combined with the destination's originally received signal through maximum ratio combining (MRC) at the destination terminal. The direct and cooperative transmission frames are described in Algorithm 1.

\section{Network Algorithm}

The transmission of signals from source to destination can either be direct transmission or cooperative transmission depending on whether or not the received signal reaches a threshold CQI (SNR) value. The system algorithm is given below;

Algorithm 1 - PRS Cooperative Diversity Algorithm

$S$ - Satellite; $R_{N}$ - Available Relays; $R_{B}$ - Selected Best Relay; $D$ - Destination; $C B R-$ Chosen Best Relay signal; $M R C$ - Maximum Ratio Combining; $\gamma_{s d}$ - Satellite-Destination SNR; $\gamma_{t h}-$ threshold SNR; $\hat{\gamma}_{s r d}-$ Predicted CQI (SNR) for the cooperative link.

\section{Start}

$S$ broadcasts

$D$ and $R_{N}$ receive signal (and keeps its times series in a buffer)

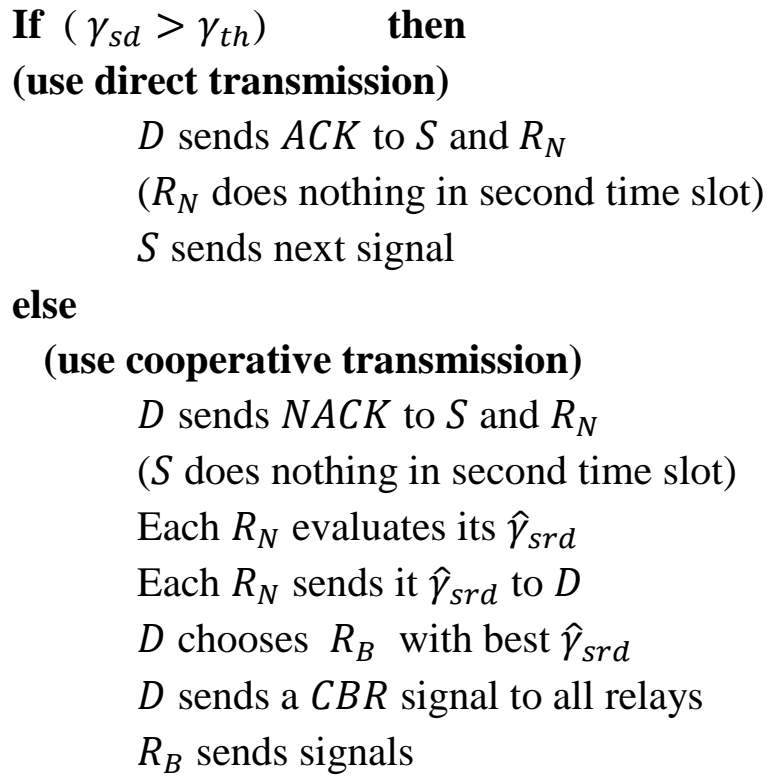

Signals combined at $D$ through $M R C$

\section{End If}

\section{End}

From Fig. 3, the problem of outdated CQI can be clearly elaborated. The relays send their estimated CQI after time $t_{2}$. The selected best relay is contacted to retransmit after time $t_{5}$. 
The time delay, $d=t_{5}-t_{2}$, between what is reported as $\mathrm{CQI}_{2}$ and what is eventually transmitted as $\mathrm{CQI}_{5}$ is enormous in satellite systems and lead to the outdated CQI problem. The diversity advantage might not be achieved if the selection of the best relay is based on $\mathrm{CQI}_{2}$ alone. The use of prediction to determine the CQI at the transmission time is used to alleviate the outdated CQI problem. The CQI value at transmission $\mathrm{CQI}_{5}$ is predicted and relay selection is now based on the best predicted $\mathrm{CQI}_{5}$ value. The applied prediction algorithms are discussed in the next section.

\section{APPLICATION OF PREDICTION ALGORITHMS TO RELAY-SELECTION COOPERATIVE DIVERSITY}

Several prediction algorithms have been developed [19] - [23]. However, due to the characteristics of the LMS system, long range prediction algorithms should be used. They must be able to predict several distance (or time frames) ahead due to the long delay in propagation for satellite networks. Two types of prediction models considered are - linear prediction and pattern-matching prediction. They have long range prediction capabilities and have shown better performance and ease of implementation as compared to other kinds of prediction models [20], [21].

\section{Linear Prediction (LP) Algorithms for the PRS Cooperation}

A linear prediction model forecasts the amplitude of a signal at time $m$, i.e. $\gamma(m)$, using a linearly weighted combination of $M$ past samples $[\gamma(m-1), \gamma(m-2), \ldots, \gamma(m-M)]$ as [22];

$$
\hat{\gamma}(m)=\sum_{k=1}^{M} a_{k} \gamma(m-k)
$$

where the integer variable $m$ is the discrete time index, $\hat{\gamma}(m)$ is the prediction of $\gamma(m), a_{k}$ is the predictor coefficient, $M$ is the auto-regression model order (or number of past samples used in predicting the next sample). The AR prediction coefficients $a_{k}$ can be computed by several algorithms, e.g. the Levinson-Durbin Recursive algorithm employed in [22]. The LP algorithms; Minimum Mean Square Error (MMSE) and Weighted Least Square Error (WLSE) are employed in this work because they have the lowest prediction error $e(m)$, defined as the difference between the actual sample value $\gamma(m)$ and its predicted value $\hat{\gamma}(m)$.

\section{A.1 Minimum Mean Square Error Linear Prediction (MMSE-LP) Algorithm}

The MMSE linear prediction is obtained by minimizing the mean square error criterion defined as

$$
E\left[e^{2}(m)\right]=E\left[\left(\gamma(m)-\sum_{k=1}^{M} a_{k}^{(D)} \gamma(m-k)\right)^{2}\right]
$$


where $a_{k}^{(D)}$ is the predictor coefficient, $D$ is the number of steps ahead for which the prediction is to be made and $E[$.$] is the expectation function. The least mean square$ (LMS) error solution gives the Weiner-Hopf equation, $R a^{(D)}=r$ where $a^{(D)}$ is the predictor coefficient, $r$ is the autocorrelation vector and $R$ is the autocorrelation function matrix of the input vector. The algorithm for MMSE linear predication used for the PRS cooperative diversity is given in Algorithm 2.

Algorithm 2 - Linear Prediction, MMSE approach [22]

The algorithm is initialized by setting $\hat{a}_{0}=1, P_{0}=R_{\gamma \gamma}(0), \Delta_{0}=R_{\gamma \gamma}(1)$.

For $m=1$ to $M$ (where $M$ is the order of prediction)

1. Calculate $m t h$ order reflection coefficient given by

$$
\Gamma_{m}=-\frac{\Delta_{m-1}}{P_{m-1}}
$$

where $P_{m}$ is the $m$ th order filter

2. Calculate the coefficients $\hat{a}_{m, k}$ for the $m t h$ order prediction-error filter, given by

$$
\hat{a}_{m, k}=\hat{a}_{m-1, k}+\Gamma_{m} \hat{a}_{m-1, m-k,}^{*} \quad k=0,1, \ldots, m
$$

where,

$$
\hat{a}_{M, k}=\left\{\begin{array}{cc}
1 & k=0 \\
-a_{M, k} & k=1,2, \ldots, M
\end{array}\right.
$$

$\hat{a}_{m-1, m-k,}^{*}$ is the conjugate of $\hat{a}_{m-1, m-k}$

3. Calculate the Root Mean Square (RMS) error for the $m t h$ order filter as

$$
P_{m}=P_{m-1}\left(1-\left|\Gamma_{m}\right|^{2}\right)
$$

4. Calculate $\Delta_{m}$, given by

$$
\Delta_{m}=r_{m}^{B T} a_{m-1}
$$

where,

$r_{m}^{B T}=\left[\begin{array}{llll}R_{\gamma \gamma}(m) & R_{\gamma \gamma}(m-1) & \ldots & R_{\gamma \gamma}(1)\end{array}\right]$ and

$R_{\gamma \gamma}(m)$ denotes the autocorrelation function $(E[\gamma(m) \gamma(m-k)])$ of the sequence $\gamma(m)$ for a lag $k$.

\section{A.2 Weighted Least Square Error Linear Prediction (WLSE-LP) Algorithm}

The WLSE algorithm bases its prediction on minimizing the weighted sum of the error taken for a given set of weights. In its algorithm, new sets of filter coefficients are found at each 
time $t=m$ and using those coefficients, the value of the coefficient for the next instance of time $t=m+1$ is predicted. The major advantage of the WLSE algorithm in comparison with the MMSE algorithm is that the autocorrelation function of the input process is not required for the WLSE algorithm. The formula for the WLSE algorithm is given as;

$$
\hat{\gamma}(m)=\frac{1}{2} \sum_{i=1}^{M} \alpha_{i}\left[a_{M}^{T} u(i)-\gamma(i)\right]^{2}
$$

where $\hat{\gamma}(m)$ is the best linear unbiased estimator, $\alpha_{i}$ are the weights, $a_{M}^{T}$ is the transpose of the coefficient vector and $u(i)$ is the input to the filter at time $t=i$ i.e. $u(i)=[\gamma(m-$ 1) $\gamma(m-2) \ldots \gamma(m-M)]^{T}$ and $\gamma(m)=a_{M}^{T}(m-1) u(m)$.

The algorithm for WLSE - LP is given in Algorithm 3.

Algorithm 3 - Linear Prediction WLSE approach [22]

The algorithm is started with $a_{M}=[1,0,0, \ldots, 0]^{T}$ and $P(1)=I$, the $M \times M$ identity matrix. Hence, the next samples of the input process are adaptively estimated.

$M$ is the order of prediction; $P$ is the complex square matrix with every principal minor $>0$; $\alpha$ is the forgetting factor chosen to be 0.99

For $m=2$ to $\infty$ (prediction starts at time 2 and can extend to any range)

1. Calculate the current predicted output $\hat{\gamma}(m)=a_{M}^{T}(m-1) u(m)$

2. Update the coefficient vector

$$
a_{M}(m)=a_{M}(m-1)+\frac{P(m-1) u(m)}{\alpha+u^{T}(m) P(m-1) u(m)}[\gamma(m)-\hat{\gamma}(m)]
$$

3. Update the $P$ matrix

$$
P(m)=\frac{1}{\alpha}\left\{P(m-1)-\frac{P(m-1) u(m) u^{T}(m) P(m-1)}{\alpha+u^{T}(m) P(m-1) u(m)}\right\}
$$

\section{Pattern-Matching Prediction Algorithm for the PRS Cooperation}

Pattern-matching prediction models are formed by observing past signal values and seeking to develop patterns based on those measurements. By assuming that these patterns are repetitive, future values of signal are thus obtained. The main advantage of pattern-matching prediction over other methods of prediction (like linear prediction) is that unlike other predictions methods, it does not make specific assumptions about the noise, fading or interference process [23].

The pattern-matching prediction algorithm developed for the PRS cooperative diversity scheme is given in Algorithm 4. 
For each available cooperative relay terminal;

1. Take CQI measurements at time interval $T_{s}$ to form a time series $c_{o}, c_{1}, c_{2}, \ldots, c_{n}, \ldots$ where $c_{n}$ is the CQI estimate at time $\left(n \times T_{s}\right)$

2. Filter the signal samples to eliminate inherent noise. A low pass filter is used to generate the filtered signal given as

$$
s_{n}=\alpha c_{n}+(1-\alpha) s_{n-1}
$$

where $s_{n}$ is the filtered (smoothed) CQI estimate at time $\left(n \times T_{s}\right), \alpha$ is the forgetting factor chosen to be 0.99 . Thus, $s_{n}=s_{0}, s_{1}, s_{2}, \ldots, s_{n}$ for $n=0,1,2, \ldots, n$. This value is then taken as the training data and is stored in a buffer by each receiver (or relay terminal). $s_{n}=s_{0}, s_{1}, s_{2}, \ldots, s_{n}$ is therefore equivalent to the input signals $[\gamma(m-$ 1), $\gamma(m-2), \ldots, \gamma(m-M)]$ of the linear prediction algorithms.

3. At query time, collect the query order $q$ and the prediction order $p$.

4. Form a query or current lag by taking the last $q$ measurements in the training data, i.e.,

$$
\text { query }=\left\{s_{n-q+1}, s_{n-q+2}, \ldots, s_{n}\right\}
$$

5. Form the lags or windows using the remaining part of the training data. Each lag must have the same size as the query order. Hence;

$$
\begin{aligned}
& \operatorname{lag} 1=\left\{s_{n-2 q+1}, s_{n-2 q+2}, \ldots, s_{n-q}\right\} \\
& \operatorname{lag} 2=\left\{s_{n-3 q+1}, s_{n-3 q+2}, \ldots, s_{n-2 q}\right\} \\
& \operatorname{lag} 3=\left\{s_{n-4 q+1}, s_{n-4 q+2}, \ldots, s_{n-3 q}\right\}
\end{aligned}
$$

Take all possible lags from the available training data in the buffer up to a lag $m$ to form a series of lags $i ; i=1,2,3, \ldots, m$. Lag $m$ is given as

$$
\operatorname{lag} m=\left\{s_{n-(m+1) q+1}, s_{n-(m+1) q+2}, \ldots, s_{n-m q}\right\}
$$

$m$ is chosen as a tradeoff between accuracy of the prediction algorithm and the use of available memory of each relay terminal.

6. Find the normalized cross correlation $\rho_{i}$ of the current lag with each lag $i ; i=$ $1,2,3, \ldots, m$

The normalized cross-correlation $\rho_{i}$ formula, given two series $x(i)$ and $y(i)$ where $i=1,2,3, \ldots, m$ is given as 


$$
\rho_{i}=\frac{\sum_{i=1}^{m}[(x(i)-\bar{x})(y(i)-\bar{y})]}{\sqrt{\sum_{i=1}^{m}(x(i)-\bar{x})^{2}} \sqrt{\sum_{i=1}^{m}(y(i)-\bar{y})^{2}}}
$$

7. Determined the lag with the highest normalized cross-correlation, $\max \left(\rho_{i}\right)$. This lag with the highest cross correlation is called the match lag.

8. Divide $p$ by $q$ to determine the set $(x, y)$ of the prediction, where $x$ is quotient of the division (and is also the number of lags ahead of the match lag needed to determine the prediction value(s)) and $y$ is the remainder of the division (and is the number of steps in the prediction lag that gives the predicted value(s), the last lag of $x$ being the prediction lag).

9. Return the value(s) in the prediction lag as the predicted $C Q I$ value(s).

\section{PERFORMANCE ANALYSIS}

The performance of the developed PRS cooperative diversity scheme is investigated in terms of outage probability and the average bit error probability.

\section{A. Outage Probability}

The outage probability $P_{0}$ is defined as the probability that the end-to-end Signal to Noise ratio (SNR) i.e. the received SNR at the output of the MRC, $\hat{\gamma}_{M R C}$, will fall below a certain predefined threshold SNR $\gamma_{t h}$;

$$
P_{0}=P\left[\hat{\gamma}_{M R C}<\gamma_{t h}\right]
$$

where the output SNR $\hat{\gamma}_{M R C}$ is given as [24],

$$
\hat{\gamma}_{M R C}=\gamma_{s d}+\min \left(\hat{\gamma}_{s r}, \hat{\gamma}_{r d}\right)
$$

and $\gamma_{s d}$ is the source-destination (S-D) SNR, $\hat{\gamma}_{s r}$ is the predicted source-relay (S-R) SNR, $\hat{\gamma}_{r d}$ is the predicted relay-destination (R-D) SNR and $\min \left(\hat{\gamma}_{s r}, \hat{\gamma}_{r d}\right)$ is the minimum of $\hat{\gamma}_{s r}$ and $\hat{\gamma}_{r d}$.

The wireless channel is modeled as a two-state (good or bad) LMS channel for the different possible combinations of the S-R-D links. The links S-R, R-D and combined S-R-D can fall into either of the states at a given time leading to eight different combinations $A_{j}, \quad j=$ $1,2, \ldots, 8$ occurring as summarized in Table 1 . Each of the eight states generates different probabilities. 


\section{TABLE 1}

Different possible combinations for the S-R-D link

\begin{tabular}{|c|c|c|c|c|}
\hline $\begin{array}{l}\text { Cooperative link (S- } \\
\text { R-D) }\end{array}$ & $\begin{array}{l}\text { State; } \mathrm{G} \text { is } \\
\text { good, } \mathrm{B} \text { is } \\
\text { Bad }\end{array}$ & $\begin{array}{l}\text { Probability of total } \\
\text { (S-R-D) link being } \\
\text { G }\end{array}$ & $\begin{array}{l}\text { State; } \mathrm{G} \text { is } \\
\text { good, } \mathrm{B} \text { is } \\
\text { Bad }\end{array}$ & $\begin{array}{l}\text { Probability of } \\
\text { total (S-R-D) } \\
\text { link being } B\end{array}$ \\
\hline S-D & $G$ & \multirow[b]{2}{*}{$A_{1}$} & $\bar{G}$ & \multirow[b]{2}{*}{$A_{5}$} \\
\hline R-D & $\mathrm{G}$ & & $\mathrm{G}$ & \\
\hline S-D & B & \multirow[b]{2}{*}{$A_{2}$} & B & \multirow[b]{2}{*}{$A_{6}$} \\
\hline R-D & $\mathrm{G}$ & & $\mathrm{G}$ & \\
\hline S-D & $\mathrm{G}$ & \multirow[b]{2}{*}{$A_{3}$} & $\mathrm{G}$ & \multirow[b]{2}{*}{$A_{7}$} \\
\hline R-D & B & & B & \\
\hline S-D & B & \multirow{3}{*}{$A_{4}$} & B & \multirow{3}{*}{$A_{8}$} \\
\hline R-D & B & & B & \\
\hline & & & & \\
\hline
\end{tabular}

Let $P\left(A_{j}\right)$ be the probability of $A_{j}$ occurring. The outage probability for the two-state LMS cooperative diversity system is therefore given as;

$$
P_{o}=\sum_{j=1}^{8}\left[P\left\{\hat{\gamma}_{M R C} \leq \gamma_{t h}\right\} P\left(A_{j}\right)\right]
$$

This is determined for the various cooperative schemes as follows.

\section{Amplify and Forward (AF)}

The outage probability of the PRS cooperative diversity for the LMS system with Amplifyand-Forward (AF) is;

$$
P_{o}=\sum_{j=1}^{8}\left[P\left\{\gamma_{s d} \leq \gamma_{t h}\right\} P\left\{\gamma_{s d}+\hat{\gamma}_{s r d} \leq \gamma_{t h} \mid \gamma_{s d}<\gamma_{t h}\right\} P\left(A_{j}\right)\right]
$$


This reduces to;

$$
P_{o}=\sum_{j=1}^{8}\left[P\left\{\hat{\gamma}_{M R C} \leq \gamma_{t h}\right\} P\left(A_{j}\right)\right]
$$

The PDF of $\hat{\gamma}_{M R C}, f_{\widehat{\gamma}_{M R C}}(\gamma)$ is;

$$
f_{\widehat{\gamma}_{M R C}}(\gamma)=\int_{-\infty}^{\infty} f_{\gamma_{s d}}\left(\gamma_{1}\right) f_{\widehat{\gamma}_{s r d}}\left(\gamma_{2}\right) d \gamma_{2}
$$

where $f_{\gamma_{s d}}\left(\gamma_{1}\right)$ is the PDF of the SNR for the S-D link and $f_{\widehat{\gamma}_{s r d}}\left(\gamma_{2}\right)$ is the PDF of $\min \left(\hat{\gamma}_{s r}, \hat{\gamma}_{r d}\right)$. The CDF of the SNR for the link, $F_{\widehat{\gamma}_{M R C}}(\gamma)$ is;

$$
F_{\widehat{\gamma}_{M R C}}(\gamma)=\int_{0}^{\infty} \int_{0}^{\gamma_{t h}-\widehat{\gamma}_{s r d}} f_{\gamma_{s d}}\left(\gamma_{1}\right) f_{\widehat{\gamma}_{s r d}}\left(\gamma_{2}\right) d \gamma_{1} d \gamma_{2}
$$

Hence, the outage probability $P_{o}$ becomes;

$$
P_{o}=\sum_{j=1}^{8}\left[\int_{0}^{\infty} \int_{0}^{\gamma_{t h}-\widehat{\gamma}_{s r d}} f_{\gamma_{s d}}\left(\gamma_{1}\right) f_{\widehat{\gamma}_{s r d}}\left(\gamma_{2}\right) d \gamma_{1} d \gamma_{2} \times P\left(A_{j}\right)\right]
$$

The various PDFs and CDFs of the links are calculated next. For the source-destination (S-D) link, the Loo's model (Rayleigh-Lognormal distributions) is employed. The PDF of the S-D link $f_{\gamma_{s d}}\left(\gamma_{1}\right)$ is [26],

$$
f_{\gamma_{s d}}\left(\gamma_{1}\right)=\frac{r}{\sigma_{1} \sqrt{2 \pi d_{0}}} \int_{0}^{\infty} \frac{1}{z} \exp \left[-\frac{(\ln z-\mu)^{2}}{2 d_{0}}-\frac{\left(r^{2}+z^{2}\right)}{2 \sigma_{1}}\right] \cdot I_{0}\left(\frac{r z}{\sigma_{1}}\right) d z
$$

and its CDF is

$$
F_{\gamma_{s d}}\left(\gamma_{1}\right)=1-\int_{0}^{\infty} Q_{1}\left(\frac{y}{\sqrt{\sigma_{1}}}, \frac{R}{\sqrt{\sigma_{1}}}\right) \frac{1}{y \sqrt{2 \pi d_{0}}} \exp \left(-\frac{(\ln \mathrm{y}-\mu)^{2}}{2 d_{0}}\right) d y
$$

where $\sigma_{1}$ is the average power of the multipath scattering, $\mu$ is the mean of the direct component, $d_{0}$ is the variance of the direct component $\ln z$, and $I_{0}($.$) is the zeroth order$ modified Bessel function of the first kind, $R$ is the threshold signal amplitude and $Q_{1}(a, b)=$ $\int_{b}^{\infty} z \cdot \exp \left(-\frac{z^{2}+a^{2}}{2}\right) \cdot I_{0}(a z) d z$ represents the first-step Marcum function.

For the source-relay-destination (S-R-D) link, the predicted SNR between S-R, $\hat{\gamma}_{s r}$ is represented as a Corazza's model (Rician-Lognormal distributions) and the predicted SNR between R-D, $\hat{\gamma}_{r d}$ is represented as Rayleigh distribution. The prediction algorithms of section III generate the probabilities of the predicted SNR using the predicted mean, average power and standard deviation of the various distributions. Hence, the PDF of S-R is given as [27], 


$$
f_{\widehat{\gamma}_{s r}}\left(\gamma_{2}\right)=\frac{r}{\sqrt{2 \pi} \hat{\sigma}_{2}^{2} \hat{\sigma}_{3}} \cdot \int_{0}^{\infty} \frac{1}{y^{3}} \exp \left(-\frac{(r / y)^{2}+\hat{\rho}^{2}}{2 \hat{\sigma}_{2}^{2}}-\frac{\left(\ln y-\widehat{m}_{3}\right)^{2}}{2 \hat{\sigma}_{3}^{2}}\right) \cdot I_{0}\left(\frac{r \hat{\rho}}{y \hat{\sigma}_{2}^{2}}\right) d y
$$

and its CDF is,

$$
\begin{array}{r}
F_{\widehat{\vartheta}_{s r}}\left(\gamma_{2}\right)=\frac{2(\widehat{K}+1)}{\hat{\sigma}_{3} \sqrt{2 \pi}} \cdot \exp (-\widehat{K}) \int_{0}^{R} r \int_{0}^{\infty} \frac{1}{y^{3}} \exp \left(-\frac{(\widehat{K}+1) r^{2}}{y^{2}}\right. \\
\left.-\frac{\left(\ln y-\widehat{m}_{3}\right)^{2}}{2 \hat{\sigma}_{3}^{2}}\right) \cdot I_{0}\left(\frac{2 r \sqrt{\widehat{K}(\widehat{K}+1)}}{y}\right) d y d r
\end{array}
$$

where $\hat{\sigma}_{2}^{2}$ is the predicted average power of the multipath scattering, $\hat{\rho}$ is the predicted amplitude of the direct component, $\widehat{m}_{3}$ is the predicted mean of the direct component and $\hat{\sigma}_{3}$ is the predicted standard deviation of the direct component and $I_{0}($.$) is the zeroth order$ modified Bessel function of the first kind. The Rician factor $\widehat{K}$ is given as $\widehat{K}=\hat{\rho}^{2} /\left(2 \hat{\sigma}_{2}^{2}\right)$ and the received signal power is normalized i.e. $\hat{\rho}^{2}+2 \hat{\sigma}_{2}^{2}=1$.

The PDF of the predicted relay-destination (R-D) link is the Raleigh distribution which is,

$$
f_{\widehat{\gamma}_{r d}}\left(\gamma_{2}\right)=\frac{r}{\hat{\sigma}_{3}^{2}} \exp \left(-r^{2} / 2 \hat{\sigma}_{3}^{2}\right)
$$

and its $\mathrm{CDF}$ is,

$$
F_{\widehat{\gamma}_{r d}}\left(\gamma_{2}\right)=1-\exp \left(-r^{2} / 2 \hat{\sigma}_{3}^{2}\right)
$$

where $r$ is the received signal envelop and $\hat{\sigma}_{3}^{2}$ is the predicted average power for the multipath scattering. To obtain $f_{\widehat{\gamma}_{s r d}}\left(\gamma_{2}\right)$, the law of probability for independent distributions is employed. Let $F_{\widehat{\gamma}_{s r d}}\left(\gamma_{2}\right)$ be the CDF of $f_{\widehat{\gamma}_{s r d}}\left(\gamma_{2}\right)$. Following statistical analysis for independent distributions, $F_{\widehat{\gamma}_{s r d}}\left(\gamma_{2}\right)$ is [28],

$$
F_{\widehat{\gamma}_{s r d}}\left(\gamma_{2}\right)=F_{\widehat{\gamma}_{s r}}\left(\gamma_{2}\right)+F_{\widehat{\gamma}_{r d}}\left(\gamma_{2}\right)-F_{\widehat{\gamma}_{s r}}\left(\gamma_{2}\right) F_{\widehat{\gamma}_{r d}}\left(\gamma_{2}\right)
$$

$f_{\widehat{\gamma}_{s r d}}\left(\gamma_{2}\right)$ is obtained by taking the derivative of its CDF. This gives;

$$
f_{\widehat{\gamma}_{s r d}}\left(\gamma_{2}\right)=\left(1-f_{\widehat{\gamma}_{s r}}\left(\gamma_{2}\right)\right)\left(1-F_{\widehat{\gamma}_{r d}}\left(\gamma_{2}\right)\right)+\left(1-F_{\widehat{\gamma}_{s r}}\left(\gamma_{2}\right)\right)\left(1-f_{\widehat{\gamma}_{r d}}\left(\gamma_{2}\right)\right)
$$

\section{Decode and Forward $(D F)$}

The outage probability of the PRS cooperative diversity for the LMS system with Decodeand-Forward (DF) is, 


$$
P_{o}=\sum_{j=1}^{8}\left[\left(P\left\{\hat{\gamma}_{s r} \leq \gamma_{t h}\right\} P\left\{\gamma_{s d} \leq \gamma_{t h}\right\}+P\left\{\hat{\gamma}_{s r}>\gamma_{t h}\right\} P\left\{\hat{\gamma}_{s r}+\hat{\gamma}_{r d} \leq \gamma_{t h}\right\}\right) \times P\left(A_{j}\right)\right]
$$

Assuming that $\hat{\gamma}_{s r}$ and $\hat{\gamma}_{r d}$ are independent and their sum $X=\hat{\gamma}_{s r}+\hat{\gamma}_{r d}$, the CDF of $X$, $F_{X}(x)$, is given by,

$$
F_{X}(x)=\int_{-\infty}^{\infty} P\left\{\hat{\gamma}_{s r}+\hat{\gamma}_{r d} \leq \gamma_{t h} \mid \hat{\gamma}_{s r}=\gamma_{1}\right\} f_{\widehat{\gamma}_{s r}}\left(\gamma_{1}\right) d \gamma_{1}
$$

where $f_{\widehat{\gamma}_{s r}}\left(\gamma_{1}\right)$ is the PDF of $\hat{\gamma}_{s r}$. This can be simplified to,

$$
F_{X}(x)=\int_{-\infty}^{\infty} F_{\widehat{\gamma}_{r d}}\left(\gamma_{t h}-\gamma_{1}\right) f_{\widehat{\gamma}_{s r}}\left(\gamma_{1}\right) d \gamma_{1}
$$

The PDF of $X, f_{X}(x)$ is obtained as,

$$
f_{X}(x)=\int_{-\infty}^{\infty} f_{\widehat{\gamma}_{r d}}\left(\gamma_{t h}-\gamma_{1}\right) f_{\widehat{\gamma}_{s r}}\left(\gamma_{1}\right) d \gamma_{1}
$$

The outage probability $P_{o}$ for DF can thus be obtained from the formulas.

\section{B. Bit Error Probability}

The average unconditional error probability $P(e)$ of the combined signal at destination (after MRC) for the PRS cooperation using either the AF or the DF schemes is given by [24],

$$
P(e)=\sum_{j=1}^{8}\left[P\left(\gamma_{s d} \leq \gamma_{t h}\right) \times P_{\text {coop }}(e)+\left(1-P\left(\gamma_{s d} \leq \gamma_{t h}\right) \times P_{d i r}(e) P\left(A_{j}\right)\right]\right.
$$

where $\gamma_{s d}$ is the instantaneous SNR between $\mathrm{S}$ and $\mathrm{D}, \gamma_{t h}$ is the threshold SNR, $P_{\text {coop }}(e)$ is the average probability that an error occurs in the combined S-R-D link, $P_{d i r}(e)$ is the average probability that an error occurs at the direct (S-D) link given that the destination already decided that the relay should not forward source signal, $P\left(\gamma_{s d} \leq \gamma_{t h}\right)$ is the CDF of the S-D link. The conditional error probability $P_{d i r}(e \mid \gamma)$ for the S-D link is defined as,

$$
P_{d i r}(e \mid \gamma)=a \times \operatorname{erfc}\left(\sqrt{b \gamma_{s d}}\right)
$$

where $(a, b)$ are constants depending on the type of modulation (for the LMSS in consideration, the modulation scheme employed is the Quadrature Phase Shift Keying (QPSK) and its constant values are $a=0.5, b=1$ ), $\operatorname{erfc}(x)$ is the complimentary error function defined as,

$$
\operatorname{erfc}(x)=(2 / \sqrt{\pi}) \int_{x}^{\infty} \exp \left(-x^{2}\right) d x
$$

The average error probability for the S-D link, $P_{\text {dir }}(e)$ is therefore given as, 


$$
P_{d i r}(e)=\int_{0}^{\infty} P_{d i r}(e \mid \gamma) f_{\gamma_{s d}}\left(\gamma \mid \gamma_{s d}>\gamma_{t h}\right) d \gamma
$$

where $P_{d i r}(e \mid \gamma)$ is the conditional error probability and $f_{\gamma_{s d}}\left(\gamma \mid \gamma_{s d}>\gamma_{t h}\right)$ is the conditional PDF of $\gamma_{s d}$ given that $\gamma_{s d}$ is greater than $\gamma_{t h}$. The conditional PDF $f_{\gamma_{s d}}\left(\gamma \mid \gamma_{s d}>\gamma_{t h}\right)$ can be obtained from the PDF $f_{\gamma_{s d}}(\gamma)$.

For the Amplify-and-Forward (AF) scheme, let $P_{\text {coop_AF }}(e)$ be the average probability that an error occurs in the combined S-R-D link when AF cooperation is employed. $P_{\text {coop_AF }}(e)$ is,

$$
P_{\text {coop_AF }}(e)=a \int_{0}^{\infty} f_{\widehat{\gamma}_{M R C}}\left(\gamma \mid \gamma_{s d} \leq \gamma_{t h}\right) \operatorname{erf} c(\sqrt{b \gamma}) d \gamma
$$

where $f_{\widehat{\gamma}_{M R C}}\left(\gamma \mid \gamma_{s d} \leq \gamma_{t h}\right)$ is the conditional PDF for $f_{\widehat{\gamma}_{M R C}}(\gamma)$ conditioned on $\gamma_{s d} \leq \gamma_{t h}$. The conditional PDF $f_{\widehat{\gamma}_{M R C}}\left(\gamma \mid \gamma_{s d} \leq \gamma_{t h}\right)$ is obtained from the PDF $f_{\widehat{\gamma}_{M R C}}(\gamma)$.

For the Decode-and-Forward (DF) let $P_{c o o p_{-} D F}(e)$ be the average probability that an error occurs in the combined S-R-D link when DF cooperation is employed. $P_{\text {coop_DF }}(e)$ is,

$$
P_{\text {coop_DF }}(e)=P_{s r}(e) P_{x}(e)+\left(1-P_{s r}(e) P_{y}(e)\right)
$$

where $P_{s r}(e)$ is the probability of error at the relay, $P_{x}(e)$ is the probability of error at destination given that the relay decoded unsuccessfully and $P_{y}(e)$ is the probability of error at destination given that the relay decoded successfully. The probability of error at the relay $P_{s r}(e)$ is,

$$
P_{s r}(e)=a\left(1-\sqrt{\frac{b \bar{\gamma}_{s r}}{1+b \bar{\gamma}_{s r}}}\right)
$$

If there is a decision error at the relay, the relay forwards an erroneous signal to the destination. The error probability due to error propagation $P_{x}(e)$ has been bounded with the worst value $P_{x}(e)<0.5$ [24].

In the case of spatial diversity being achieved (i.e., the relay decodes correctly), there is still a probability of an error occurring at the destination and that probability is given by $P_{y}(e)$. To find $P_{y}(e)$, we re-employ the combined S-R-D link for decode-and-forward given by $X=\hat{\gamma}_{s r}+\hat{\gamma}_{r d}$ and its PDF $f_{X}(x)$. Hence, the average error probability $P_{y}(e)$ is written as,

$$
P_{y}(e)=\int_{0}^{\infty} f_{X}\left(x \mid \gamma_{s d} \leq \gamma_{t h}\right) \operatorname{erfc}(\sqrt{b x}) d x
$$

where $f_{X}\left(x \mid \gamma_{s d} \leq \gamma_{t h}\right)$ is the conditional PDF for $f_{X}(x)$ conditioned on $\gamma_{s d} \leq \gamma_{t h}$. $f_{X}\left(x \mid \gamma_{s d} \leq \gamma_{t h}\right)$ is obtained from $f_{X}(x)$. 


\section{ANALYTICAL AND SIMULATION RESULTS}

The performance of the Predictive Relay Selection (PRS) cooperative diversity in comparison with the direct communication and outdated cooperative communication models is presented in this section. A two-state LMS Markov chain model developed in [25] is employed in generating the faded signal. The parameters for the steady state probability matrix $\mathrm{W}$ and the state transition matrix $\mathrm{P}$ for the LMS model as well as values for the average multipath power, mean and standard deviation used in generating the faded signals for the good and bad states are also as in [25]. Both urban and rural areas at elevation angle $60^{\circ}$ are considered. For the simulation, ten (10) relays were assumed to be available within the destination terminal's interference range out of which a single best was selected. The plots of the performance in terms of outage probability $P_{0}$ and bit error probability (BER) for the cooperative diversity schemes are presented. The extensive performance results presented in this work features the three prediction algorithms considered compared with the outdated and non-cooperation (direct) communication. The developed analytical model was also validated by simulation.

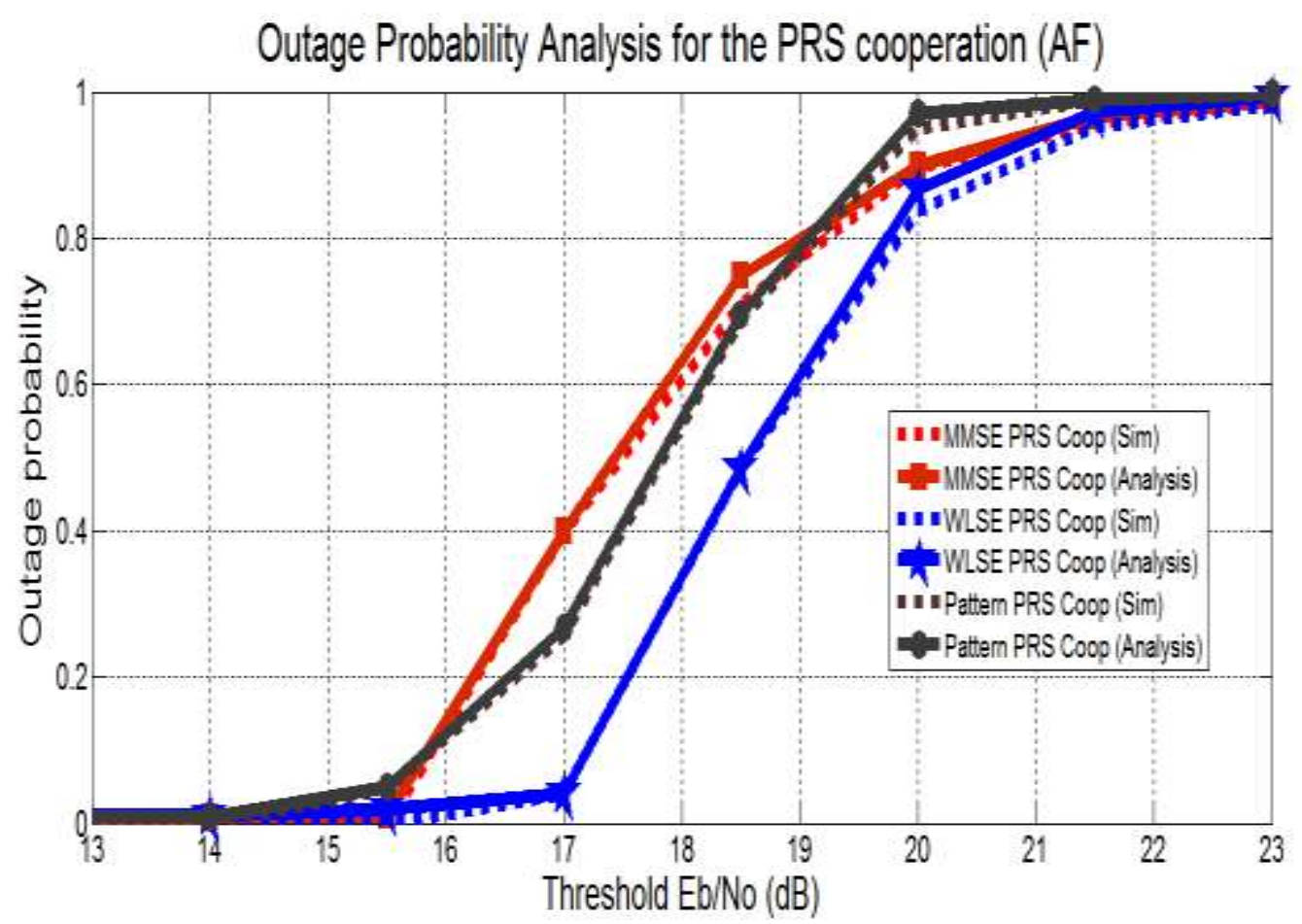

Fig. 4. Outage probability performance for the different PRS cooperative models using Amplify-and-Forward Scheme

The simulation and analytical results of the outage probability $\left(P_{0}\right)$ versus threshold SNR of the three predictive relay-selection (PRS) cooperative communications are shown in Fig. 4. and Fig. 5. for Amplify-and-Forward (AF) and Decode-and-Forward (DF) schemes respectively. The results indicate that the developed analytical model is well matched and validated by the simulation. It also shows that the outage probability generally increases with increasing threshold SNR. At thresholds below $14 \mathrm{~dB}$, the outage probability is very low (approximately zero). At thresholds between $16 \mathrm{~dB}$ and $21 \mathrm{~dB}$, the outage probability gradually increases until it reaches unity and saturates. The reason is that at a higher threshold SNR demand for a given communication QoS, the likelihood of an outage is usually more 
prominent. The WLSE linear prediction model outperforms the other prediction models for both the AF and the DF schemes.

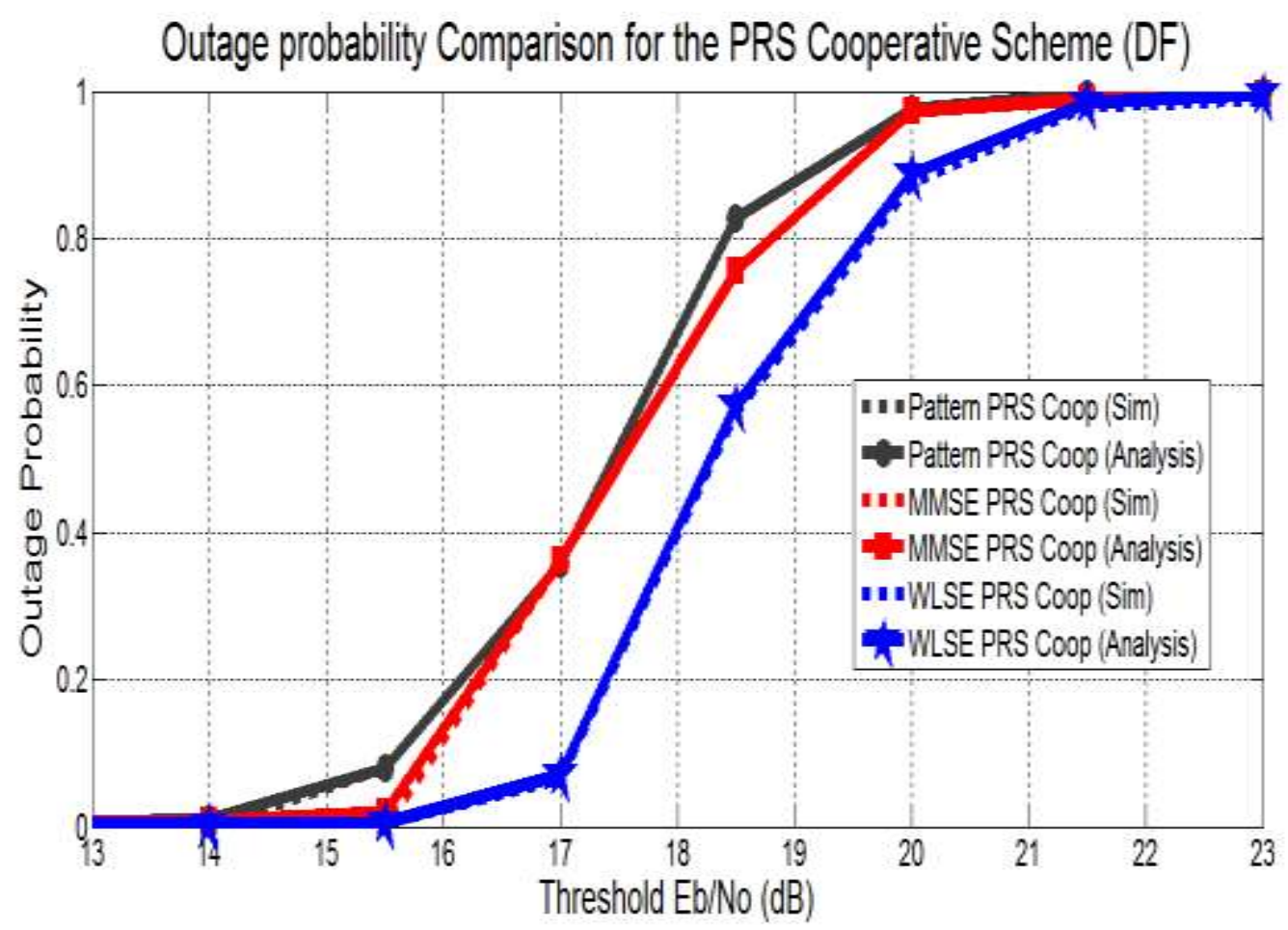

Fig. 5. Outage probability performance for the different PRS cooperative models using Decode-and-Forward Scheme

The simulation and analytical results of the average bit error probability versus average SNR $\left(\mathrm{E}_{\mathrm{b}} / \mathrm{N}_{\mathrm{o}}\right.$ in $\left.\mathrm{dB}\right)$ of the three predictive relay-selection (PRS) cooperative communications are shown in Fig. 6. and Fig. 7. for Amplify-and-Forward (AF) and Decode-and-Forward (DF) schemes respectively. The developed analytical model is also well matched and validated by the simulation. The BER reduces with an increasing SNR value while the WLSE linear prediction model also outperforms the other prediction models for both the AF and the DF protocols. From the outage probability and the bit error probability results, it can be concluded that the WLSE predictive cooperation is the best predictive model for LMSS cooperative diversity. 


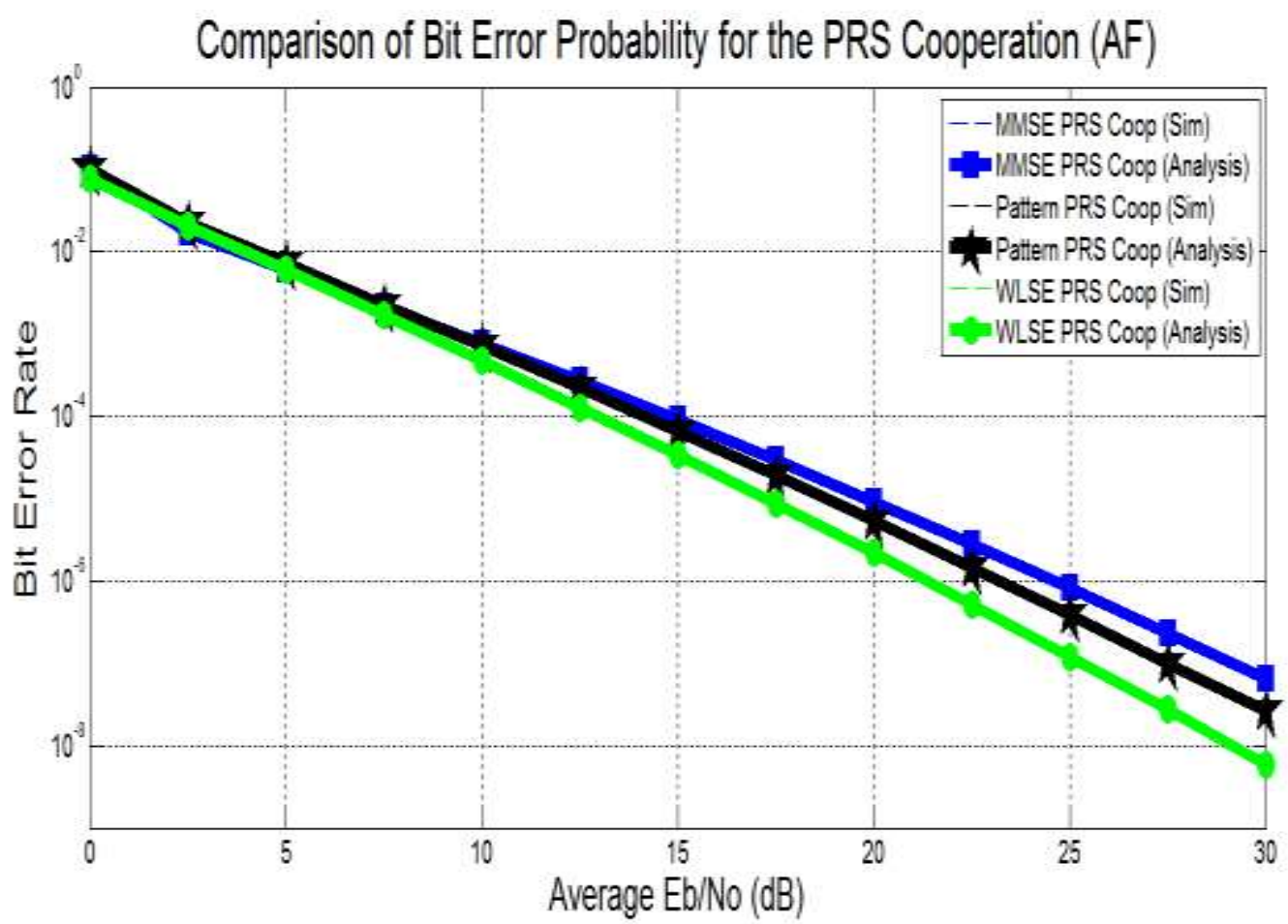

Fig. 6. Bit error probability performance for the different PRS cooperative models using Amplify-and-Forward Scheme

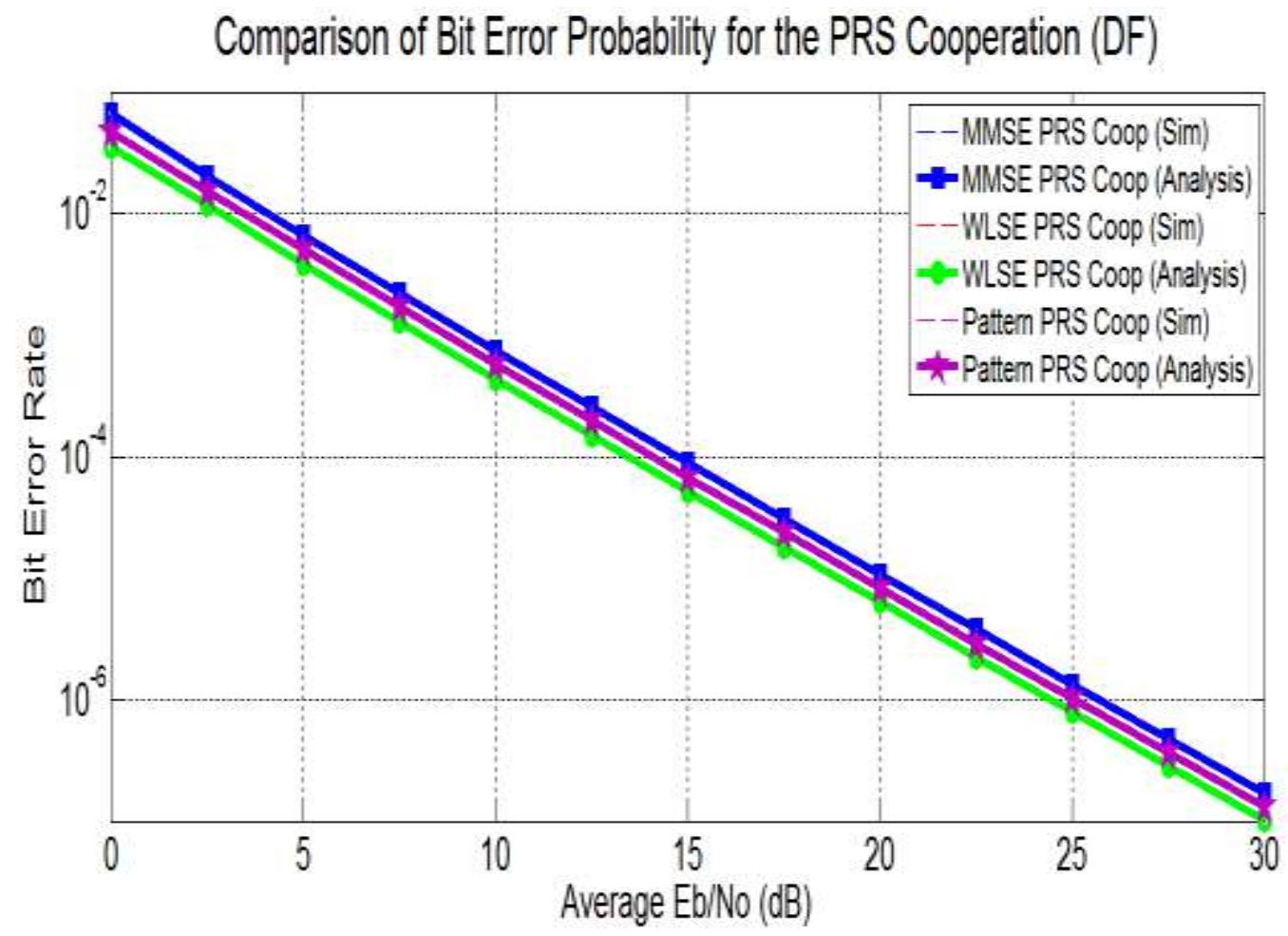

Fig. 7. Bit error probability performance for the different PRS cooperative models using Decode-and-Forward Scheme

The outage probability comparison for the cooperative communication and direct communication (non-cooperation) is shown in Fig. 8 - Fig.10. Both predictive cooperation and outdated cooperation are considered for all three predictive algorithms studied. At low SNR values, the performance of the direct and cooperative communication is similar. This is 
because cooperation is only employed when the direct communication is impossible which is most improbable at low SNRs. As the SNR increases, it is observed that the outage experienced during cooperative communication is significantly less than the outage experienced for direct communication(for instance, at a threshold $10 \mathrm{~dB}$, while the direct communication outage probability is above 0.9 , the cooperative communication outage probability was still approximately 0 ). This is expected as the cooperative system gives an average SNR value greater than the direct system at every instance. Furthermore, all PRS cooperative diversity protocols outperformed the outdated CQI cooperation. This result is significant in that it confirms that the relay-selection cooperation using outdated CQI cannot always guarantee the intended quality of service and that the PRS cooperation gives a higher diversity advantage.

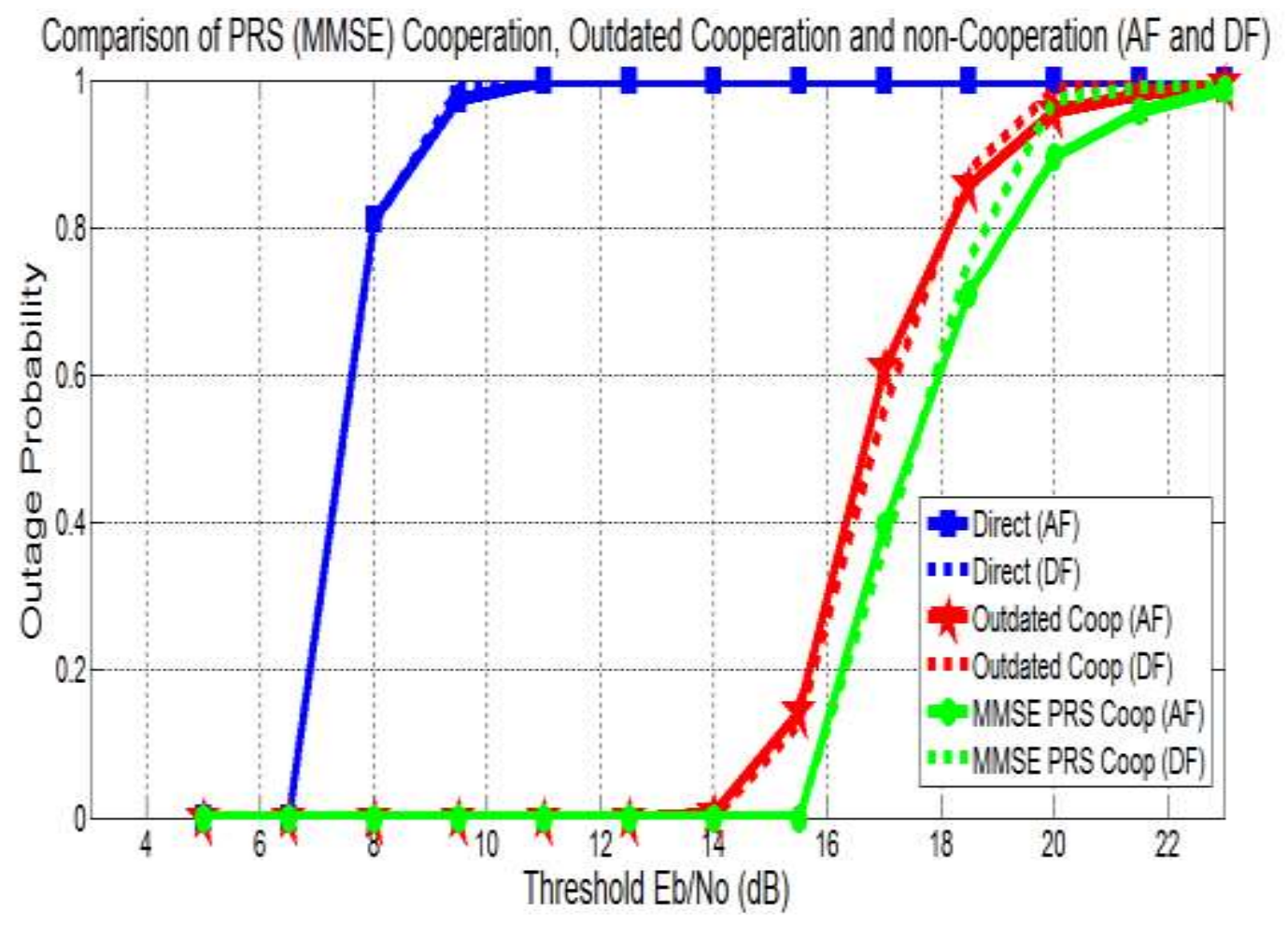

Fig. 8. Outage probability comparison of the PRS (MMSE) cooperation with outdated cooperation and direct communication 


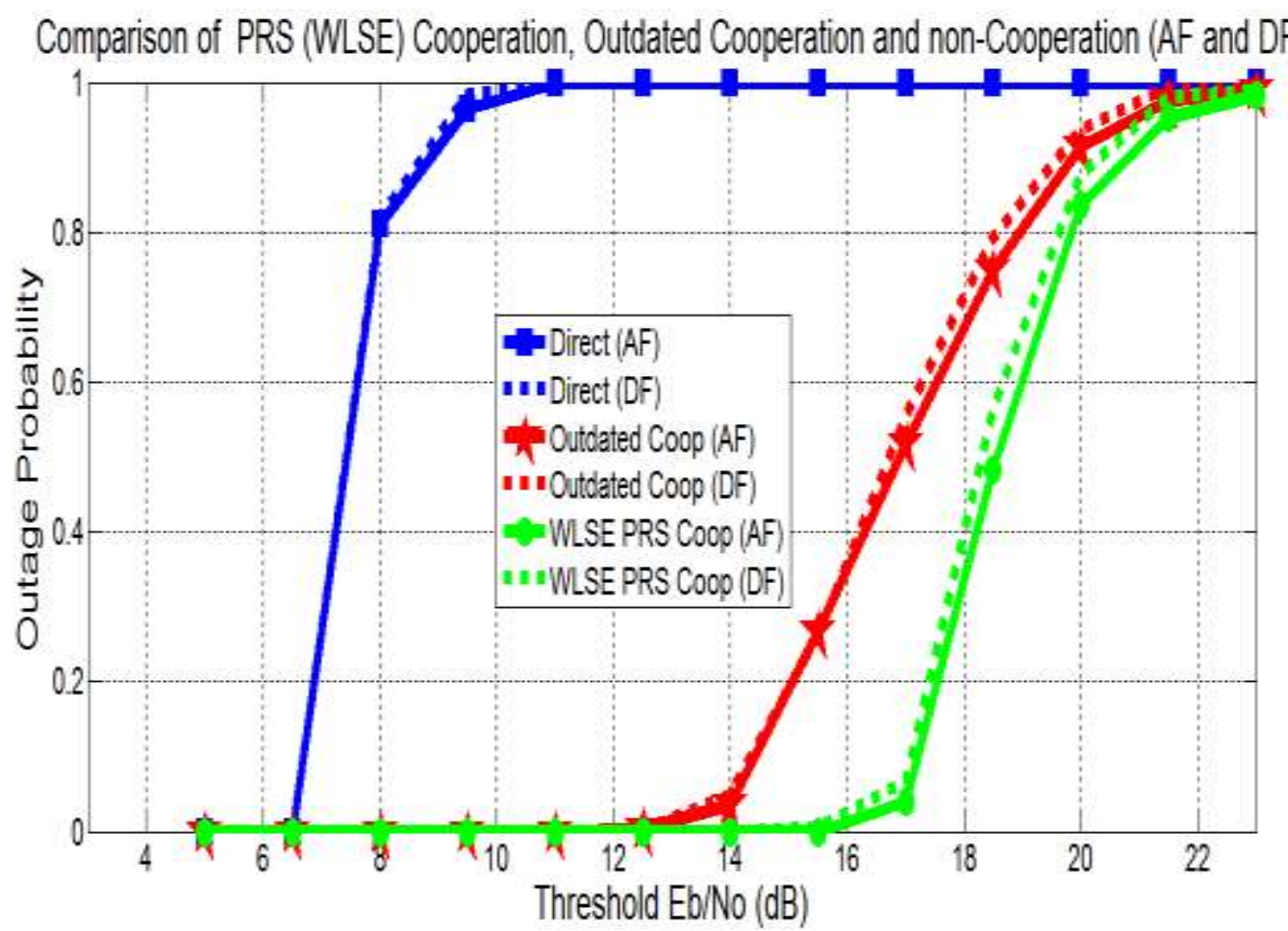

Fig. 9. Outage probability comparison of the PRS (WLSE) cooperation with outdated cooperation and direct communication

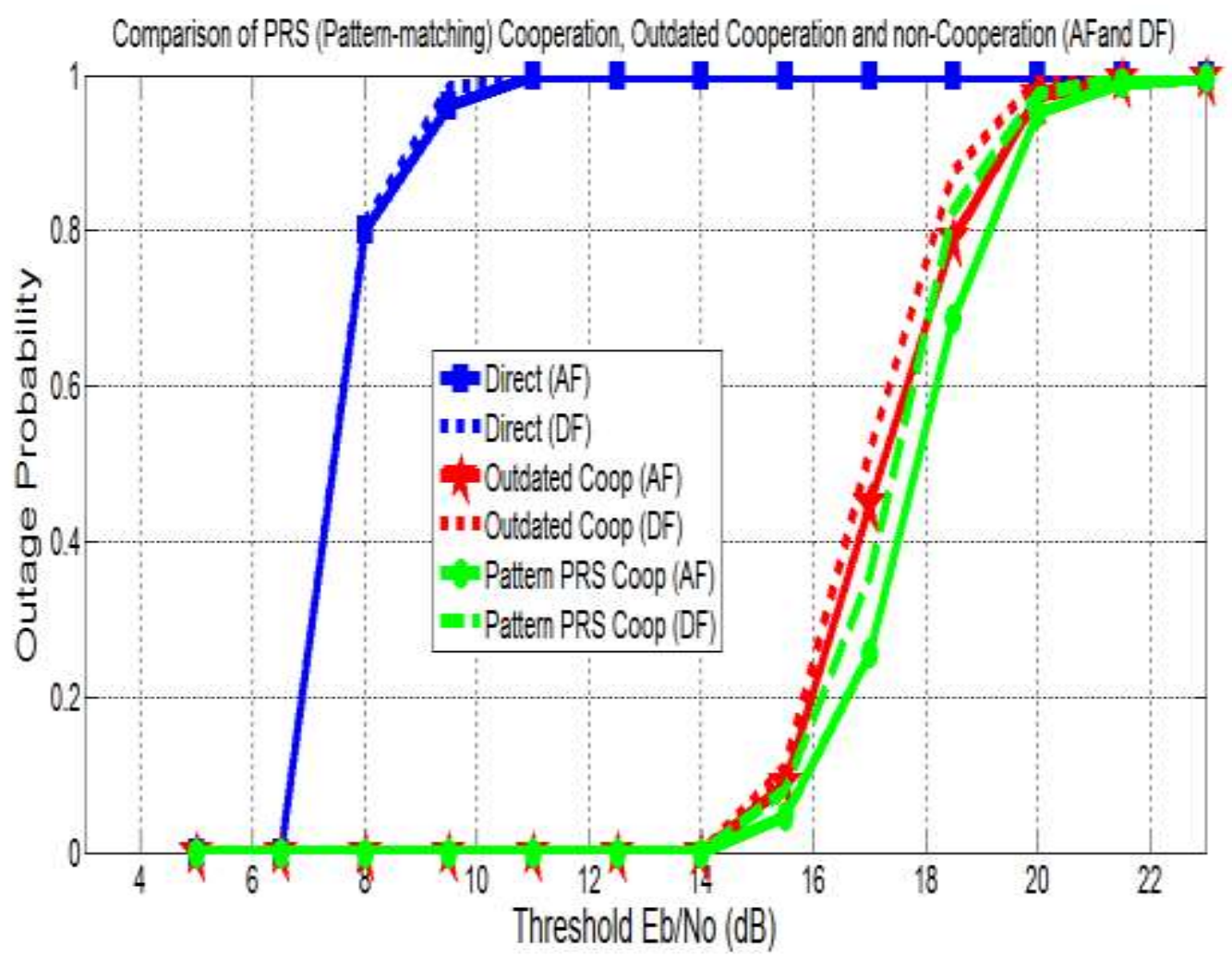

Fig. 10. Outage probability comparison of the PRS (Pattern-matching) cooperation with outdated cooperation and direct communication

The outage probability is compared for multiple relay selection in Fig. 11. From the results, it can be observed that the more the relays employed in the cooperation, the better the outage performance. This is simply because as more relays are being selected to transmit their 
signals, the overall signal at the destination improves thus reducing the probability of an outage, although at a higher signalling cost [13].

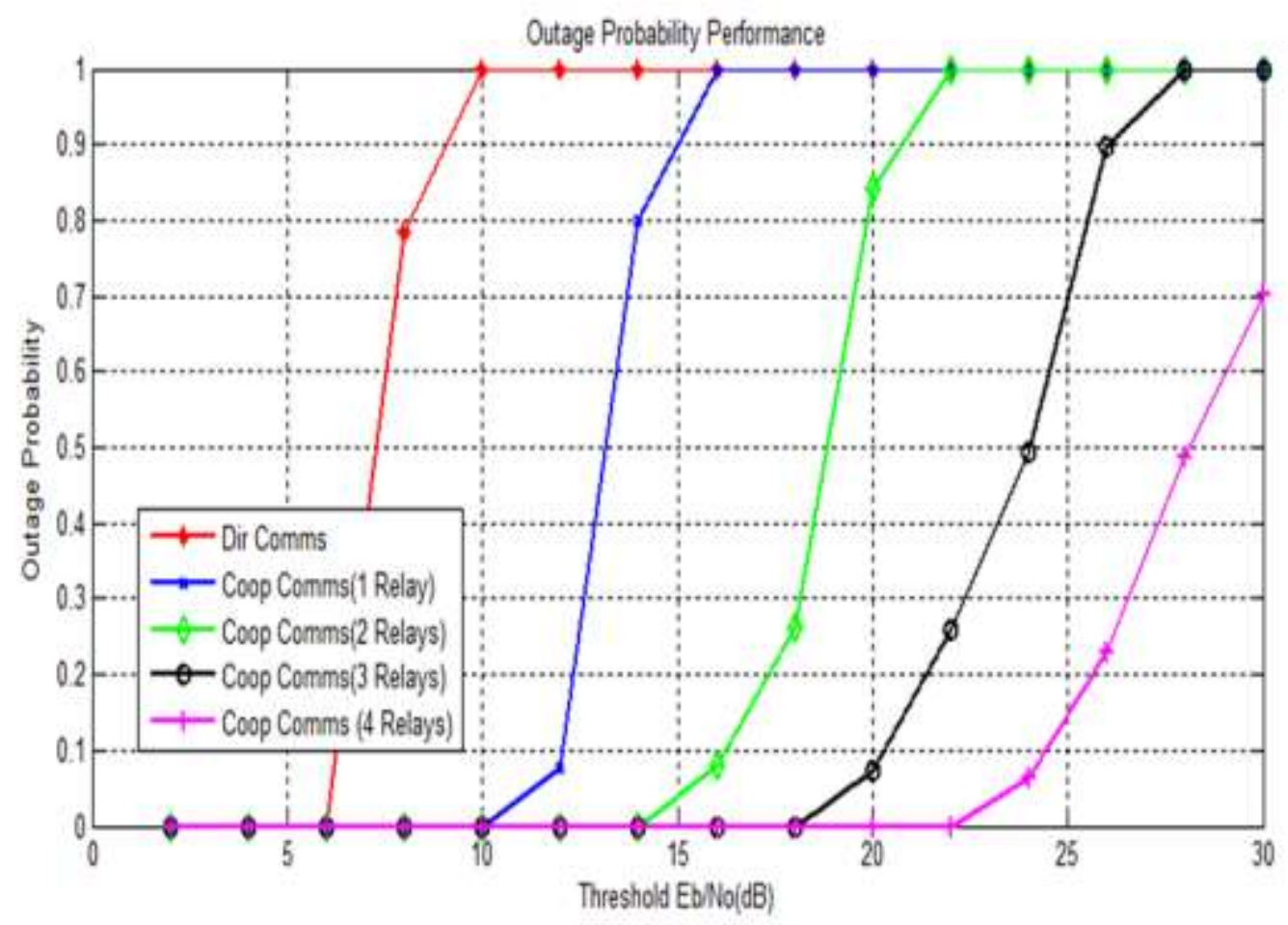

Fig. 11. Outage Probability compared when multiple relays are selected during cooperative communication

The bit error rate (BER) plots of the PRS cooperation, outdated cooperation and direct communication are compared in Fig. 12. to Fig. 14. for both the AF and DF cooperative schemes. Similar to the results for the outage probability, the three PRS cooperative schemes outperformed both the direct communication as well as the outdated CQI cooperation. From the outage probability and bit error probability results, it can be easily concluded that the PRS cooperative diversity performs better than either direct communication (non-cooperation) or cooperation with outdated cooperative diversity. 


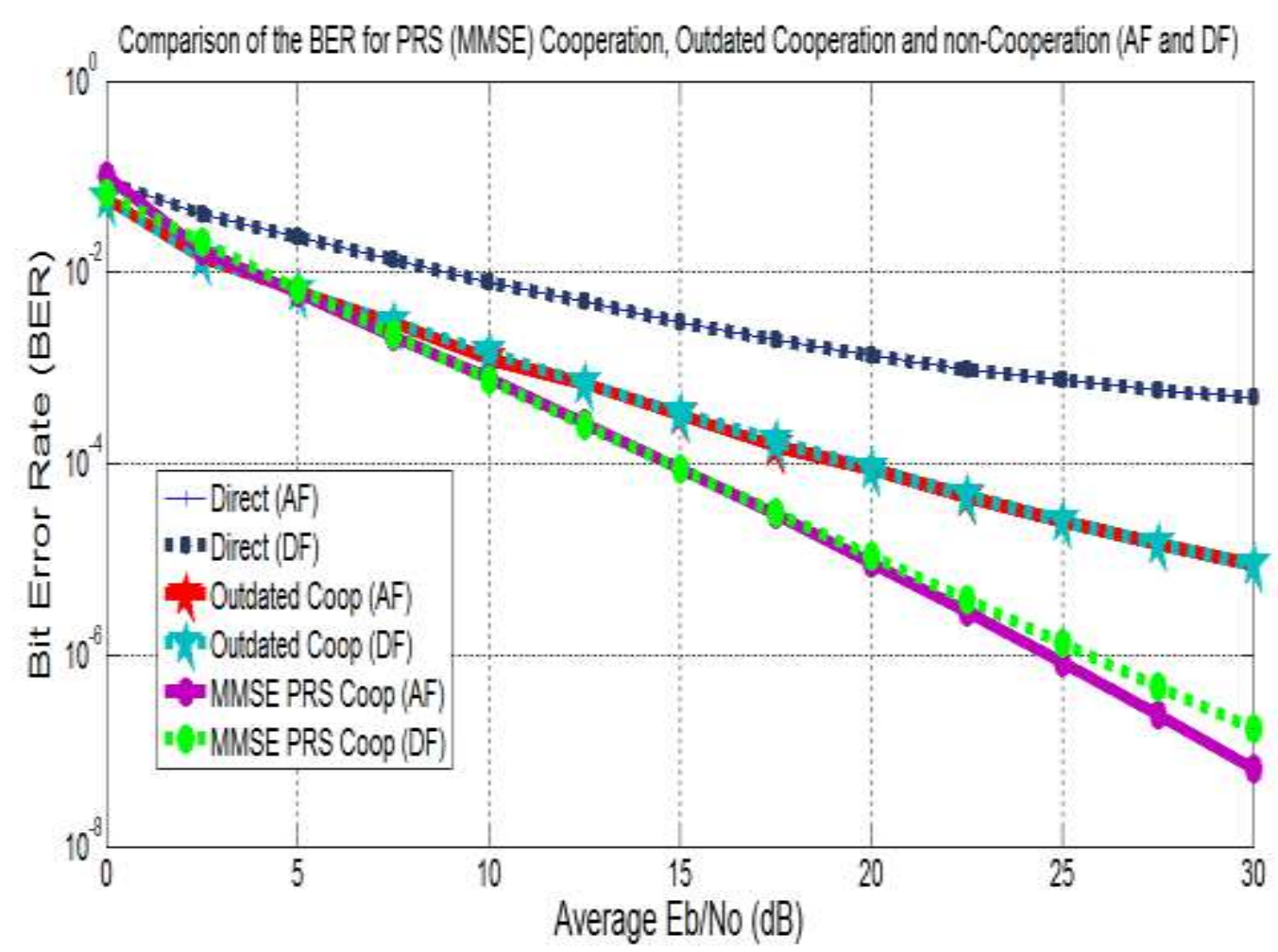

Fig. 12. Bit Error probability comparison of the PRS (MMSE) cooperation with outdated cooperation and direct communication

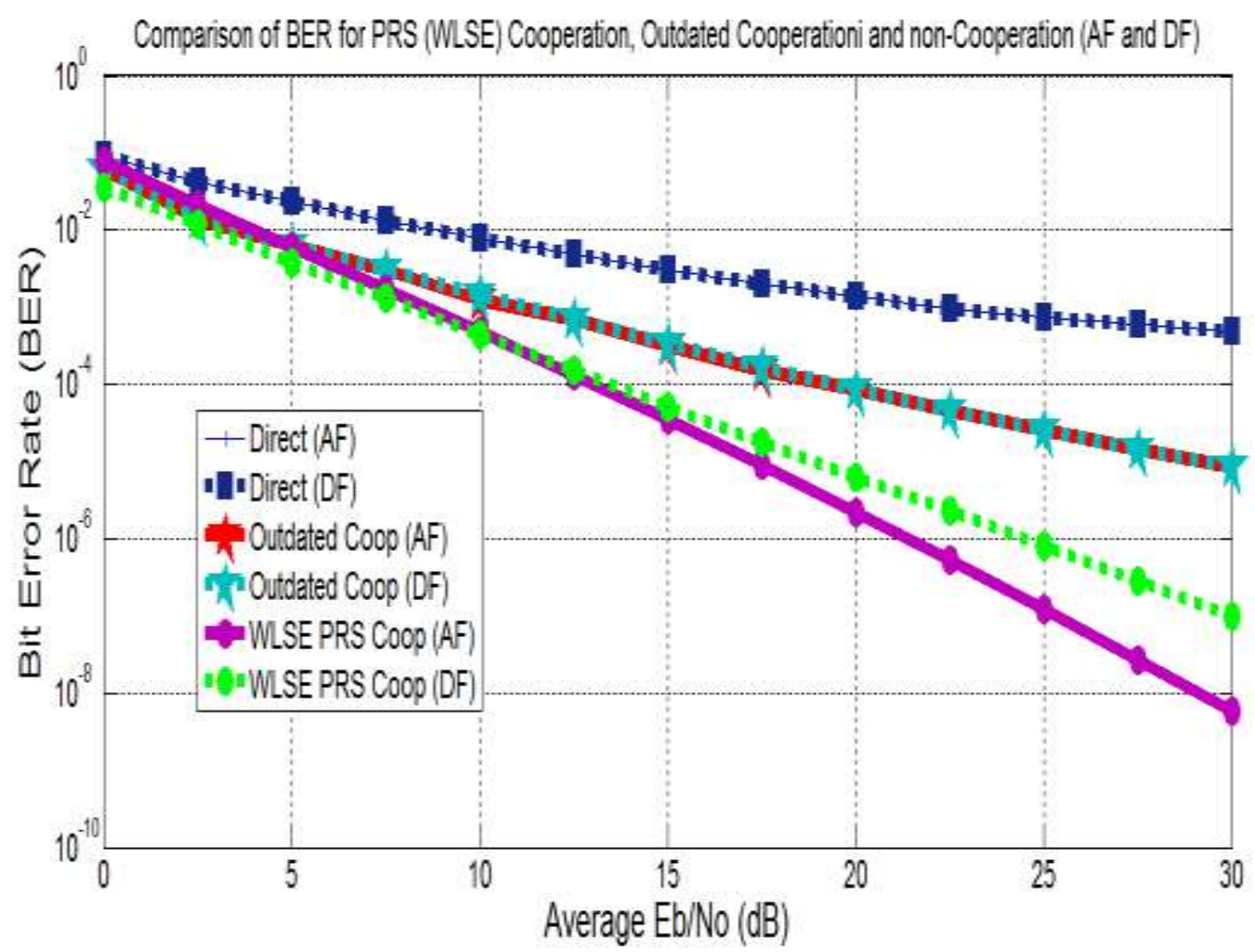

Fig. 13. Bit Error probability comparison of the PRS (WLSE) cooperation with outdated cooperation and direct communication 


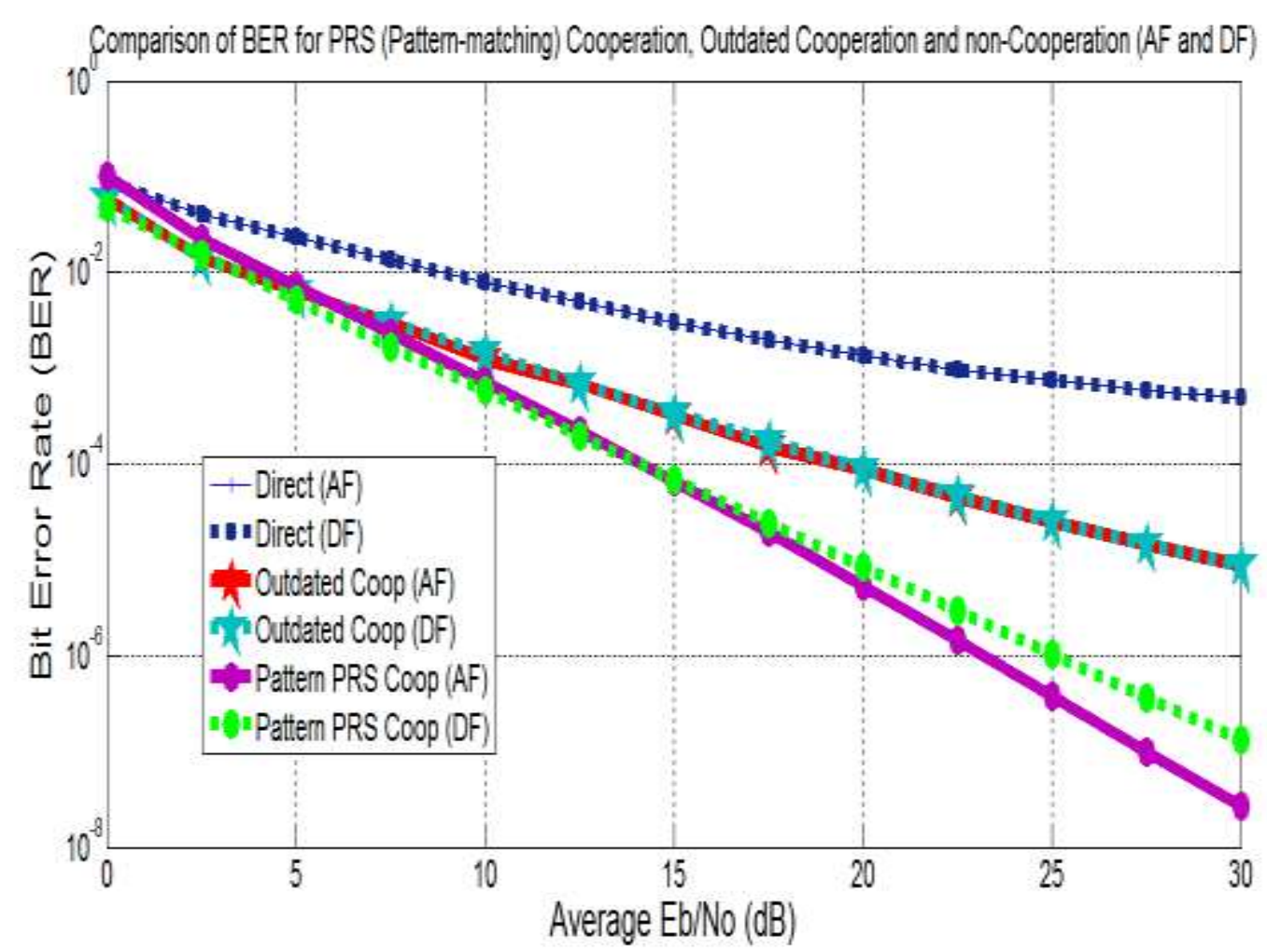

Fig. 14. Bit Error probability comparison of the PRS (Pattern-matching) cooperation with outdated cooperation and direct communication

Finally, the performance of the three PRS cooperative schemes for two different environment types (rural and urban which gives the extreme cases of environment types) is investigated. The results of the outage probability and the bit error probability are shown in Fig. 15. and Fig. 16. respectively. The results show that both outage probability and bit error probability are generally better for the rural environment than for the urban environment. This is because the fading effects are higher in the urban than in the rural environment, thus making the probability of an outage in the urban environment greater. Overall, the WLSE linear prediction algorithm showed best performance in terms of outage probability and bit error probability (for both AF and DF schemes) as compared to the MMSE linear prediction and the pattern-matching prediction schemes in both environment types. The reason for the best performance of the WLSE linear prediction is that it adaptively changes its coefficients in order to meet the minimum WLSE criterion. In computational complexity however, the pattern-matching prediction model is a lot less easy than the linear prediction models in that it simply makes patterns from past measurements and makes prediction based on these past measurements. 


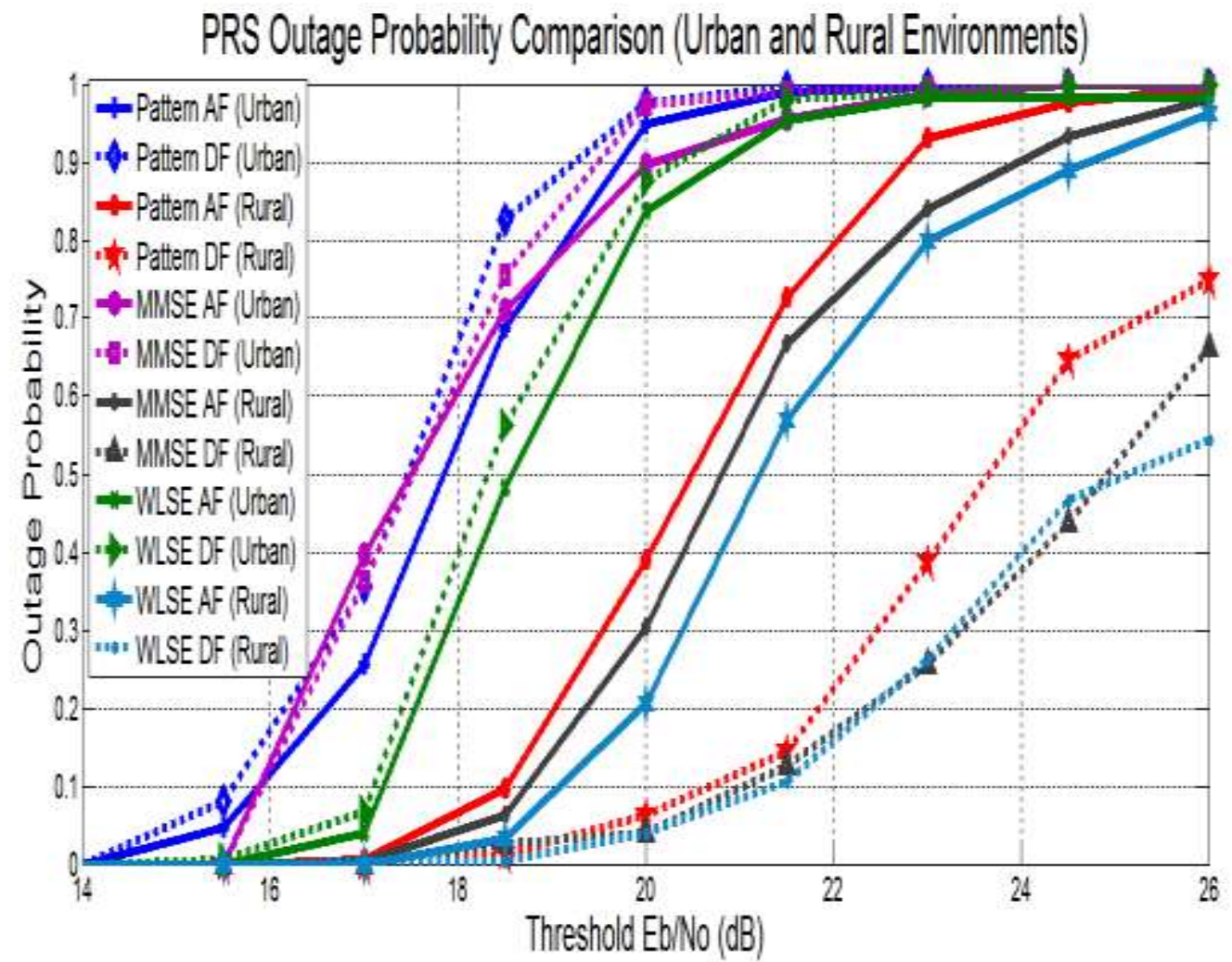

Fig. 15. Outage Probability comparison of the PRS cooperation diversity schemes for the rural and the urban environments using both AF and DF cooperation

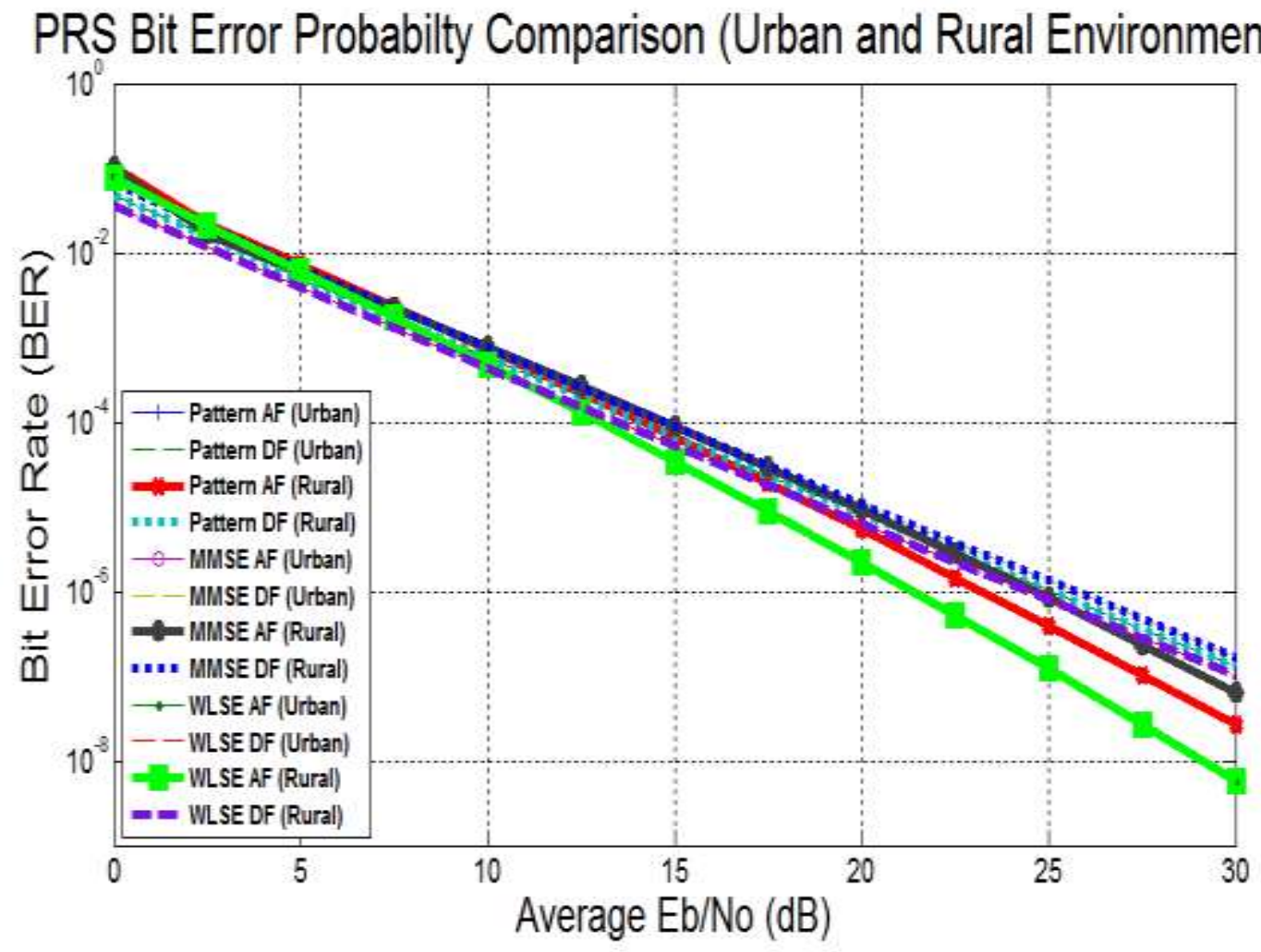

Fig. 16. Bit Error Probability comparison of the PRS cooperation diversity schemes for the rural and the urban environments using both AF and DF cooperation 


\section{CONCLUSION}

The effects of both mobility and the long propagation delay are a major limitation to the effectiveness of cooperative diversity in LMS communication systems. In this research work, a novel Predictive Relay-Selection (PRS) cooperative diversity scheme for LMS systems was developed to curtail the effect of user mobility and long propagation delay. Prediction algorithms were employed in determining the future channel qualities of the available relay terminals to determine the best relay for selection. The performance of the PRS cooperative diversity scheme in terms of outage probability and bit error probability were presented with the PRS cooperative scheme performing better than both the direct communication and the cooperative communication using outdated CQI. Of the three predictive schemes considered, the WLSE linear prediction cooperative scheme performed best in AF and DF cooperation as well as in rural and urban environment types. The PRS cooperative diversity scheme as investigated is designed for future wireless communication. With the introduction of hybrid satellite-terrestrial networking, satellite mobile terminals will be readily available in close proximity with one another and can therefore act as cooperators or relays thus making the scheme's implementation feasible.

\section{REFERENCES}

1. Luca Simone Ronga and Rosalba Suffritti, "Cooperative Strategies for Satellite Access," InTechOpen book chapter, Published on: 2010-08-18

2. J. N. Laneman, N. C. Tse, and G. W. Wornell, "Cooperative diversity in wireless networks: Efficient protocols and outage behavior," IEEE Trans. Inf. Theory, vol.50, No.12, pp. 3062-3080, Dec. 2004

3. Jie Xiao; Feng Shaodong; Li Wei; Zhang Gengxin; Zang Guozhen, "Research on Cooperative Diversity in Mobile Satellite Communication System," Second International Workshop on Education Technology and Computer Science (ETCS), vol.1, no., pp.304-307, 6-7 March 2010

4. A. Nosratinia, T. Hunter, A. Hedayat, "Cooperative communication in wireless networks," IEEE Comm. Mag., vol. 42, no. 10, pp. 74-80, Oct. 2004

5. Ikki, S.S.; Ahmed, M.H., "Exact Error Probability and Channel Capacity of the Best-Relay Cooperative-Diversity Networks," Signal Processing Letters, IEEE, vol.16, no.12, pp.10511054, Dec. 2009

6. Torabi, M.; Haccoun, D.; Ajib, W., "Performance analysis of cooperative diversity with relay selection over non-identically distributed links," Communications, IET, vol.4, no.5, pp.596-605, March 262010

7. Weidong Guo; Ju Liu; Lina Zheng; Yuxi Liu; Guowei Zhang, "Performance analysis of a selection cooperation scheme in multi-source multi-relay networks," Wireless Communications and Signal Processing (WCSP), 2010 International Conference on , vol., no., pp.1-6, 21-23 Oct. 2010 
8. Iqbal, A.; Ahmed, K.M., "Outage probability analysis of multi-hop cooperative satelliteterrestrial network," Electrical Engineering/Electronics, Computer, Telecommunications and Information Technology (ECTI-CON), 2011 8th International Conference on, vol., no., pp.256-259, 17-19 May 2011

9. A. Bel, J. Vicario, and G. Seco-Granados, "The benefits of relay selection in WiMAX networks," in Proc. ICT Mobile Summit, 2008

10. J. Vicario and C. Antón-Haro, "Analytical assessment of multi-user vs. spatial diversity trade-offs with delayed channel state information, "IEEE Commun. Lett., Aug. 2006

11. Vicario, J.L.; Bel, A.; Lopez-Salcedo, J.A.; Seco, G., "Opportunistic relay selection with outdated CSI: outage probability and diversity analysis," Wireless Communications, IEEE Transactions on, vol.8, no.6, pp.2872-2876, June 2009

12. Haiyang Ding; Jianhua Ge; Benevides da Costa, D.; Zhuoqin Jiang, "Asymptotic Analysis of Cooperative Diversity Systems With Relay Selection in a Spectrum-Sharing Scenario," Vehicular Technology, IEEE Transactions on, vol.60, no.2, pp.457-472, Feb. 2011

13. Michalopoulos, D.S.; Suraweera, H.A.; Karagiannidis, G.K.; Schober, R., "Amplify-andForward Relay Selection with Outdated Channel Estimates," Communications, IEEE Transactions on, vol.60, no.5, pp.1278-1290, May 2012

14. Moyuan Chen; Liu, T.C.-K.; Xiaodai Dong;, "Opportunistic Multiple Relay Selection With Outdated Channel State Information," Vehicular Technology, IEEE Transactions on, vol.61, no.3, pp.1333-1345, March 2012

15. Hongyu Cui; Rongqing Zhang; Lingyang Song; Bingli Jiao, "Relay Selection for Bidirectional AF Relay Network With Outdated CSI," Vehicular Technology, IEEE Transactions on, vol.62, no.9, pp.4357,4365, Nov. 2013

16. Tiangao Gou; Jafar, S.A., "Optimal Use of Current and Outdated Channel State Information: Degrees of Freedom of the MISO BC with Mixed CSIT," Communications Letters, IEEE, vol.16, no.7, pp.1084,1087, July 2012

17. Godana, Bruhtesfa E.; Gesbert, David, "Coordinated beamforming in multicell networks with Channel State Information exchange delays," Personal Indoor and Mobile Radio Communications (PIMRC), 2013 IEEE 24th International Symposium on , vol., no., pp.713,718, 8-11 Sept. 2013

18. Sigen Ye; Blum, R.S.; Cimini, L.J., "Adaptive OFDM Systems With Imperfect Channel State Information," Wireless Communications, IEEE Transactions on , vol.5, no.11, pp.3255,3265, November 2006

19. Duel-Hallen, A., "Fading Channel Prediction for Mobile Radio Adaptive Transmission Systems," Proceedings of the IEEE, vol.95, no.12, pp.2299-2313, Dec. 2007 
20. Duel-Hallen A.; Hu S.; Hallen H., "Long range prediction of fading signals: enabling adaptive transmission for mobile radio channels," IEEE Signal Process. Mag., vol 17, pp. 62-75, May 2000

21. Heidari, A.; Khandani, A.K.; McAvoy, D., "Adaptive modeling and long-range prediction of mobile fading channels," Communications, IET, vol.4, no.1, pp.39-50, January 52010

22. Garg, M., "Linear prediction algorithms," Indian Institute of technology, Bombay, India, 2003

23. Dinh-Dung Luong; Gregoire, J.-C.; Dziong, Z., "Pattern-Based Channel Quality Prediction for Adaptive Coding and Modulation in Wireless Networks," Communications (ICC), 2010 IEEE International Conference, vol., no., pp.1-6, 23-27 May 2010

24. Ikki, S.S.; Ahmed, M.H., "Performance analysis of incremental-relaying cooperativediversity networks over Rayleigh fading channels," Communications, IET, vol.5, no.3, pp.337-349, Feb. 112011

25. Yang Mingchuan; Meng Fanyu; Shi Shuo; Guo Qing, "Markov Chain Based Two-State Satellite Mobile Channel Model," Vehicular Technology Conference (VTC Spring), 2011 IEEE 73rd, vol., no., pp.1-5, 15-18 May 2011

26. Chun Loo; Butterworth, J.S., "Land mobile satellite channel measurements and modeling," Proceedings of IEEE, vol.86, no.7, pp.1442-1463, Jul 1998

27. Corazza, G.E.; Vatalaro, F., “A statistical model for land mobile satellite channels and its applications to nongeostationary orbit systems," Vehicular Technology, IEEE Transactions on, vol.43, no.3, pp.738-742, Aug 1994

28. Chapter 4, Conditional Probability - Dartmouth College, Available: http://www.dartmouth.edu/ chance/teaching_aids/books_articles/probability_book/Chapter4. pdf 


\section{Authors' Biographies}

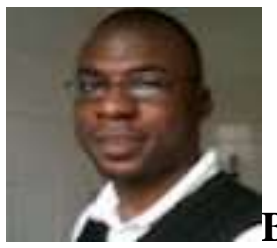

Babatunde Awoyemi received his Masters degree in Electronic Engineering from the University of KwaZulu-Natal. He is currently a Doctoral student in the department of Electrical, Electronic and Computer Engineering at the University of Pretoria. His research interest is in wireless communications with particular notch for cooperative diversity and cognitive radio networks.

Tom Walingo holds a B.Tech degree in Electrical and Communications Engineering from Moi University in Kenya, and a MScEng from the University of Natal in South Africa. He now holds a $\mathrm{PhD}$ degree in Engineering obtained at the University of Natal. He is currently with the department of Electrical, Electronic and Computer Engineering at the University of KwaZulu-Natal.

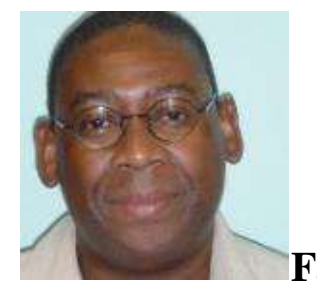

Fambirai Takawira received his BSc degree in electrical engineering (firstclass honours) from Manchester University, UK in 1981 and his PhD from Cambridge University, in 1984. He is currently a professor and the head of the School of Electrical and Information Engineering at the University of Witwatersrand, Johannesburg, South Africa. His research interests include digital communications, data networks, advanced digital transmission techniques for future generation systems and radio resource management in wireless communications. 